\title{
A Posteriori Finite Element Bounds to Linear Functional Outputs of the Three Dimensional Navier-Stokes Equations *
}

\author{
Zhong Cheng ${ }^{\dagger}$ and Marius Paraschivoiu ${ }^{\ddagger}$
}

\begin{abstract}
An implicit a posteriori finite element error estimation method is presented to inexpensively calculate lower and upper bounds for a linear functional output of the numerical solutions to the three dimensional Navier-Stokes (N-S) equations. The novelty of this research is to utilize an augmented Lagrangian based on a coarse mesh linearization of the $\mathrm{N}-\mathrm{S}$ equations and the finite element tearing and interconnecting (FETI) procedure. The latter approach extends the a posteriori bound method to the three dimensional Crouzeix-Raviart space for $\mathrm{N}-\mathrm{S}$ problems. The computational advantage of the bound procedure is that a single coupled nonsymmetric large problem can be decomposed into several uncoupled symmetric small problems. A simple model problem, which is selected here to illustrate the procedure, is to find the lower and upper bounds of the average velocity of a pressure driven, incompressible, steady Newtonian fluid flow moving at low Reynolds numbers through an endless square channel which has an array of rectangular obstacles. Numerical results show that the bounds for this output are rigorous, i.e., always in the asymptotic certainty regime, that they are sharp and that the required computational resources decrease significantly. Parallel implementation on a Beowulf cluster is also reported.
\end{abstract}

\section{Introduction}

The steady, incompressible Navier-Stokes (N-S) equations are the most commonly used mathematical model for describing the practical problems related to viscous, laminar flows. But numerical simulations of the $\mathrm{N}-\mathrm{S}$ equations require large computational resources, especially for three dimensional engineering problems. In practice, a designer using computational fluid dynamics (CFD) has to produce the most accurate result with limited computer resources. He can make a trade-off between solution accuracy and computational cost based on his experience and judgment. A posteriori error estimation methods use the calculated numerical result to assess the accuracy of the solution. These methods are becoming essential because they can help the designer to assess the accuracy and to control the simulation quality.

\footnotetext{
*accepted in International Journal for Numerical Methods in Engineering

${ }^{\dagger}$ Department of Mechanical and Industrial Engineering, University of Toronto. E-mail: zcheng@mie.utoronto.ca

${ }_{\ddagger}^{\ddagger}$ Corresponding author, Department of Mechanical and Industrial Engineering, Concordia University, H-549, 1455 de Maisonneuve Blvd. W. Montreal Quebec H3G 1M8. E-mail: paraschi@me.concordia.ca
} 
In the last decade, most of the a posteriori error estimates were focused on global error bounds in the energy norm. These works were mainly concerned with one or two dimensional problems. Among these, some works related to the approach herein include: Ainsworth and Oden developed a method to obtain the numerical error in the energy norm to estimate the accuracy of solutions of elliptic partial differential equations [1]. Verfürth [21], Bank and Welfert [2] also successfully proposed the error estimations based on the norms or seminorms associated with a solution of the Stokes equations.

Later, a posteriori error estimation techniques were extended to error metrics more closely relevant to engineering design. An engineering design often focuses on calculating the characteristic values of a system. These characteristic values are not the field solutions, such as temperature or velocities, but are design parameters, such as average temperatures, mean concentrations, drag forces or average velocities, which are termed 'outputs' herein. The error metrics of these values are much more important to a designer than the field solutions or their energy norms, because specific design objectives and optimization are generally addressed in the form of design outputs, and the design regulations are often described by lower or upper bounds to the outputs. Considering engineering design, Patera, Paraschivoiu and Peraire developed a similar procedure to that of Ainsworth and Oden for the prediction of the error in terms of bounds to design quantities, or outputs, which are the linear functionals of the field solutions. Both symmetric and nonsymmetric partial differential equations were considered $[12,16,18]$.

The proposed upper and lower bounds are obtained from the application of a constrained minimization problem formulated as an extremization of an augmented Lagrangian [16]. An output for the very large problem can be estimated quickly, inexpensively and quantitatively by calculating its rigorous bounds which are not dependent on any constraints. The bound procedure has also been extended to address the incompressible Navier-Stokes equations in two space dimensions by Machiels, Peraire, and Patera [11].

The upper and lower bounds are calculated through a hierarchical procedure on two-level finite element meshes: several global calculations on a relatively coarse mesh and many independent local calculations on subdomains related to a fine finite element mesh. The last calculation step only solves intrinsically parallel, uncoupled, symmetric local problems with interpolated hybrid-fluxes recovered as Neumann boundary conditions. The bound method can achieve very high parallel efficiency when multi-computers are exploited for these calculations. Cheng and Paraschivoiu reported that local calculations can reach more than $90 \%$ parallel efficiency in solving a two dimensional multi-material heat transfer problem on a Beowulf multiple-computer system [4].

In three space dimensions, the Ladeveze and Leguilon procedure [10] that is used to calculate the hybrid fluxes in two dimensions $[1,11,12,16,18]$ is difficult to extend. To address this drawback, Paraschivoiu reformulated the finite element tearing and interconnecting (FETI) method, which was developed by Farhat and Roux [8], for calculating bounds for the convectiondiffusion equation in three-dimensional space [17].

In this paper, the approach described in $[4,11,17]$ is extended to calculate bounds for steady, incompressible Navier-Stokes equations in three-dimensional space. The novelty of this research is to linearize the $\mathrm{N}-\mathrm{S}$ problems with respect to a coarse mesh and to exploit the resulting equations in an augmented Lagrangian. The Crouzeix-Raviart finite element space is used so that the pressure space is discontinuous and the velocities are locally divergence-free. Thus, the hybrid flux vector is decoupled to three components which can be inexpensively calculated by the FETI method. This a posteriori finite element bound procedure calculates 
bounds to the linear-functional outputs of a benchmark problem, which is a pressure driven, incompressible, steady Newtonian fluid flow moving with a low Reynolds number through an endless square channel with an array of rectangular obstacles. Since the calculation of the fine 'truth' mesh output $s_{h}$ is very expensive, the associated lower bound $s_{L B}$ and upper bound $s_{U B}$ are calculated as alternatives to $s_{h}$. The bounds offer precise and reliable information for the output $s_{h}$ at a computational cost of the same order as the coarse mesh used and with an asymptotic certainty of $s_{L B} \leq s_{h} \leq s_{U B}$. Therefore $s_{h}$ can also be estimated by the bounds:

$s_{\text {pre }}=\frac{1}{2}\left(s_{U B}+s_{L B}\right)$, which will satisfy $\left|s_{h}-s_{\text {pre }}\right| \leq \triangle$, where $\triangle=\frac{1}{2}\left(s_{U B}-s_{L B}\right)$ is the half bound gap.

The asymptotic certainty refers to the limitation that the coarse mesh must roughly resolve the flow. As shown in [11], rigorous bounds are obtained when the characteristic length of the course mesh $(H)$ is less than a threshold mesh size. To be more precise, the difference between the upper bound and the fine mesh output can be expressed as a positive term and an indefinite term. Similarly for the lower bound, the difference can be expressed as a negative and an indefinite term. It is shown [13] that the positive term converges at best like $H^{4}$ and the indefinite term as $H^{5}$ which leads to the existence of this threshold mesh size. This threshold mesh size is unknown but our numerical experience suggests that the coarse mesh size is less than the threshold mesh size even for very coarse meshes. In practice, bounds are very large for coarse meshes that barely resolve the flow and become sharper as the coarse mesh is refined. The smooth convergence of the bounds as the coarse mesh is refined ensured that bounds are in the asymptotic certainty regime.

A multiple processor implementation is developed for the FETI and fine mesh calculations. The results will be analyzed to investigate the efficiency of the bound method for parallel processing. The approach developed herein provides an inexpensive confirmation of the accuracy of a particular finite element solution to three-dimensional partial differential equations for large engineering applications.

The remainder of this paper is structured as follows. In Section 2, we review some of the key finite element fundamentals for this paper. In Section 3, we introduce a new augmented Lagrangian based on the linearized Navier-Stokes equations on the coarse mesh for the underlying N-S problem. In Section 4, we calculate the lower and upper bounds derived from the stationarity conditions of the augmented Lagrangian. Numerical results from a single processor and a Beowulf cluster are presented in Sections 5 and 6. Finally, we conclude in Section 7.

\section{Finite Element Background}

The Navier-Stokes bound procedure is based on an augmented Lagrangian. The general finite element background is reviewed first for the steady, incompressible Navier-Stokes equations, as well as for the linear output, and the discretization method.

\subsection{Problem statement and linear output}

The model problem is steady, incompressible, low Reynolds number flow driven by a forcing term in an endless square channel with an array of rectangular obstacles in the center. The fluid is assumed to be Newtonian with dynamic viscosity, $\mu$. The geometry is presented in Figure 1 where $\Omega$ is the domain and $\left(x_{1}, x_{2}, x_{3}\right)$ denotes the coordinate system, with corresponding unit 


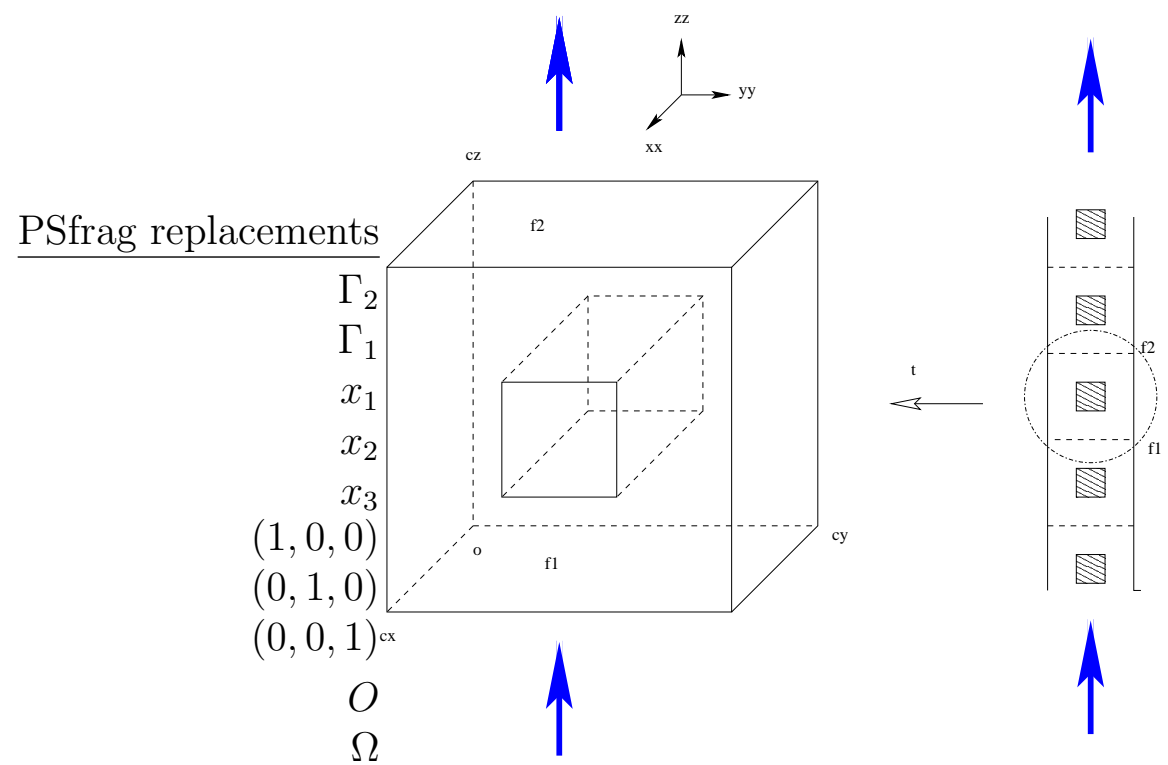

Figure 1: Geometry with homogeneous Dirichlet boundaries, except for $\Gamma_{1}$ and $\Gamma_{2}$ which have periodic boundary conditions.

vectors $\vec{x}_{1}, \vec{x}_{2}, \vec{x}_{3}$. The driving force is a pressure gradient $\frac{\Delta P}{L}$ in the $\vec{x}_{3}$ direction, where $L$ is the scaling length of the channel section, and $\Delta P$ is the pressure difference between the two reference points separated by a distance $L$ in the $\vec{x}_{3}$ direction. The fluid velocity and pressure perturbations are periodic in the $\vec{x}_{3}$ direction.

The governing equations for this incompressible low Reynolds number Navier-Stokes problem can be expressed in indicial notation as

$$
\begin{aligned}
-\frac{1}{R e} \frac{\partial^{2} u_{i}}{\partial x_{j} \partial x_{j}}+\frac{\partial\left(u_{j} u_{i}\right)}{\partial x_{j}}+\frac{\partial p}{\partial x_{i}} & =f_{i} \text { in } \Omega, \quad i=1,2,3 \\
\frac{\partial u_{i}}{\partial x_{i}} & =0 \text { in } \Omega,
\end{aligned}
$$

with boundary conditions $\left.u_{i}\right|_{\Gamma_{1}}=\left.u_{i}\right|_{\Gamma_{2}},\left.\quad p\right|_{\Gamma_{1}}=\left.p\right|_{\Gamma_{2}}$, and $u_{i}=0$ on the other boundaries. Re is the Reynolds number defined as $R e=\frac{\rho \tilde{U} \tilde{H}}{\mu}$, where $\tilde{H}$ denotes the width (or height) of the square channel and $\rho$ is the density of the fluid. The flow is assumed to be laminar with the scaling velocity $\tilde{U}$ proportional to $\tilde{U}=\frac{\tilde{H}^{2}}{c \mu} \frac{\Delta P}{L}$. Here $c$ is a constant which depends on the geometry of the channel. From [14], $c=28.45$ is selected for the square duct without obstacles. The pressure field, composed of the external driving pressure $\Delta P$ and the pressure fluctuation $p$, is non-dimensionalized by $\rho \tilde{U}^{2}$. The forcing term $f_{i}$, which is the nondimensionalized pressure gradient, is $f_{1}=f_{2}=0$ and $f_{3}=\frac{28.45}{R e}$ in the current problem. To be noted, the Reynolds number only represents the applied pressure gradient in this problem.

We define the function spaces $X=H_{0}^{1}(\Omega) \times H_{0}^{1}(\Omega) \times H_{0}^{1}(\Omega), \mathcal{Q}=L^{2}(\Omega)$ and $Y=X \times \mathcal{Q}$ including essential boundary conditions, where $L^{2}(\Omega)$ is the space of all functions which are 
square integrable over $\Omega$ and $H_{0}^{1}(\Omega)$ is the space of all functions vanishing at the essential boundary and belonging to $L^{2}(\Omega)$ whose first derivatives are also in $L^{2}(\Omega)$.

The corresponding variational form for this Navier-Stokes problem is: find $\left(u_{1}, u_{2}, u_{3}, p\right) \in$ $Y$ such that

$$
\begin{aligned}
\int_{\Omega}\left(\frac{1}{R e} \frac{\partial \omega_{i}}{\partial x_{j}} \frac{\partial u_{i}}{\partial x_{j}}-u_{i} u_{j} \frac{\partial \omega_{i}}{\partial x_{j}}-p \frac{\partial \omega_{i}}{\partial x_{i}}-f_{i} \omega_{i}\right) d V=0, \quad \forall\left(\omega_{1}, \omega_{2}, \omega_{3}\right) \in X, \\
-\int_{\Omega} r \frac{\partial u_{i}}{\partial x_{i}} d V=0, \quad \forall r \in \mathcal{Q} .
\end{aligned}
$$

Introducing discrete function spaces parameterized by $\delta$ we write $Y_{\delta}=X_{\delta} \times \mathcal{Q}_{\delta}$ for which $X_{\delta}=\operatorname{span}\left(\phi_{i}, i=1, . ., N_{v}\right)$ and $\mathcal{Q}_{\delta}=\operatorname{span}\left(\varphi_{i}, i=1, . ., N_{p}\right)$ where $N_{v}$ and $N_{p}$ are the number of nodes for each velocity component and for pressure, respectively. Discretized functions, such as each velocity component and pressure, are written:

$$
\begin{aligned}
u_{i \delta} & =\sum_{j=1}^{N_{v}} u_{i j \delta} \phi_{j} \\
p_{\delta} & =\sum_{j=1}^{N_{p}} p_{j \delta} \varphi_{j}
\end{aligned}
$$

We also introduce $\bar{u}_{i \delta}$ and $\bar{p}_{\delta}$ as the vectors of coefficients $u_{i j \delta}$ and $p_{j \delta}$, respectively.

Using this finite element discretization, equations (3) and (4) can be expressed in a general matrix form as: find $\left(u_{1 \delta}, u_{2 \delta}, u_{3 \delta}, p_{\delta}\right) \in Y_{\delta}$ that satisfies

$$
\begin{aligned}
\left(A_{\delta}+C_{\delta}\right) \bar{u}_{i \delta}+B_{i \delta}^{T} \bar{p}_{\delta} & =\bar{f}_{i \delta}, \quad i=1,2,3 \\
\sum_{i=1}^{3} B_{i \delta} \bar{u}_{i \delta} & =0
\end{aligned}
$$

where $A_{\delta}$ is the stiffness matrix associated with the diffusion term $\int \frac{1}{R e} \frac{\partial \omega_{i}}{\partial x_{j}} \frac{\partial u_{i}}{\partial x_{j}} d V, B_{i \delta}$ is associated with the pressure term $\int p \frac{\partial \omega_{i}}{\partial x_{i}} d V$ and $C_{\delta}$ is associated with the convection term $\int u_{i} u_{j} \frac{\partial \omega_{i}}{\partial x_{j}} d V$. Note that the matrix $C_{\delta}$, is computed from the velocity field $u_{i \delta}$. Details concerning weak formulations of the Navier-Stokes system may be found in [19, 20].

Engineering design is generally based on outputs which are functionals of the field solution. In this paper, only linear functionals are addressed, and bounds are calculated to the outputs, which are the average of the velocity in the $\vec{x}_{3}$ direction, $s=\int u_{3} d V$, and the corresponding matrix expression is $s_{\delta}=\bar{\ell}_{\delta}^{0^{T}} \bar{u}_{3 \delta}=1^{T} M_{\delta} \bar{u}_{3 \delta}$ with $M_{\delta}$ being the mass matrix associated with the forcing term $\int f_{i} \omega_{i} d V$. The discrete linear functional operator $\bar{\ell}_{\delta}^{0}$ is the unit vector times the mass matrix, $1^{T} M_{\delta}$.

\subsection{Newton Iterations}

The Navier-Stokes equations (1) and (2) contain a non-linear convective term $\int u_{i} u_{j} \frac{\partial \omega_{i}}{\partial x_{j}} d V$, and its associated matrix $C_{\delta}$ in equation (7) should be calculated with the velocity field $u_{i}$ with $i=1,2,3$. The Newton-Raphson nonlinear algorithm has a second-order convergence rate and 
is chosen to iteratively solve the underlying N-S equations. Gunzburger presents this method in details [9]. The algorithm is summarized below.

1. Initialization: solve the algebraic equations to obtain $\bar{u}_{1 \delta}^{0}, \bar{u}_{2 \delta}^{0}, \bar{u}_{3 \delta}^{0}, \bar{p}_{\delta}^{0}$ :

$$
\begin{aligned}
A_{\delta} \bar{u}_{i \delta}^{0}+B_{i \delta}^{T} \bar{p}_{\delta}^{0} & =\bar{f}_{i \delta}, \\
\sum_{i=1}^{3} B_{i \delta} \bar{u}_{i \delta}^{0} & =0 .
\end{aligned}
$$

2. At the $n^{\text {th }}$ step, find $\left(\bar{v}_{1 \delta}, \bar{v}_{2 \delta}, \bar{v}_{3 \delta}\right)$ and $\bar{q}_{\delta}$ that satisfy the following linear equations:

$$
\begin{aligned}
\left(A_{\delta}+D_{\delta}^{n}\right) \bar{v}_{i \delta}+\sum_{j=1}^{3} E_{i j \delta}^{n} \bar{v}_{j \delta}+B_{i \delta}^{T} \bar{q}_{\delta} & =\bar{f}_{i \delta}-\left[\left(A_{\delta}+D_{\delta}^{n}\right) \bar{u}_{i \delta}^{n}+B_{i \delta}^{T} \bar{p}_{\delta}^{n}\right] \\
\sum_{i=1}^{3} B_{i \delta} \bar{v}_{i \delta} & =-\sum_{i=1}^{3} B_{i \delta} \bar{u}_{i \delta}^{n}
\end{aligned}
$$

where the matrice $D_{\delta}^{n}$ is associated with the linearized convection terms $\int v_{i} u_{j}^{n} \frac{\partial \omega_{i}}{\partial x_{j}} d V$ and $E_{i j \delta}^{n}$ is associated with the linearized convection terms $\int u_{i}^{n} v_{j} \frac{\partial \omega_{i}}{\partial x_{j}} d V$, with $i, j=1,2,3$.

3. Set $n=n+1$ and $\left(\bar{u}_{i \delta}^{n}, \bar{p}_{\delta}^{n}\right)=\left(\bar{u}_{i \delta}^{n-1}, \bar{p}_{\delta}^{n-1}\right)+\left(\bar{v}_{i \delta}, \bar{q}_{\delta}\right)$; check the stopping criterion. If the criterion is not satisfied, update $D_{\delta}^{n}$ and $E_{i j \delta}^{n}$ with the new $u_{i \delta}^{n},(i, j=1,2,3)$, go back to Step 2 , and continue to iterate.

\subsection{Discretization meshes and function spaces}

The bound method for the Navier-Stokes equations is a two level hierarchiary numerical approximation. Two discretization meshes, $H$ for the coarse and $h$ for the fine, are considered here. The computational domain $\Omega$ is divided into a set of $k_{H}$ tetrahedra for the coarse mesh $\mathcal{T}_{H}$, and is further divided into a set of $k_{h}$ tetrahedra for the fine mesh $\mathcal{T}_{h}$ with uniform refinement $R=\frac{H}{h}$. Figure 2 illustrates the two level meshes that are used, $\mathcal{T}_{H}$ (left) and $\mathcal{T}_{h}$ (right), before the domain decomposition.

The domain $\Omega$ is also decomposed into $N_{k}$ subdomains $\Omega^{(k)}$ for both $\mathcal{T}_{H}$ and $\mathcal{T}_{h}$ so that the bound method can be exploited. For example, $\Omega$ can be decomposed into $N_{k}=24$ subdomains, $\Omega^{(k)}$, each of which is a small cube of size $\left(\frac{1}{3} \times \frac{1}{3} \times \frac{1}{3}\right)$, as shown in Figure 3 . This figure illustrates the resulting coarse and fine meshes after the domain decomposition. The thick lines represent subdomain borders.

On these two level meshes, Crouzeix-Raviart finite element spaces are used for the velocity and the pressure spaces. $X_{H}$ denotes the finite element space for the velocity on the coarse mesh and $X_{h}$ denotes the space for the velocity on the fine mesh before the domain decomposition. Also the finite element spaces for the pressure on the coarse mesh and on the fine mesh are denoted by $\mathcal{Q}_{H}$ and $\mathcal{Q}_{h}$, respectively. $\hat{X}_{H}$ and $\hat{X}_{h}$ are defined as the 'broken' velocity spaces of each subdomain $\Omega^{(k)}$ after the domain decomposition. The hybrid flux edge spaces, which are defined on the subdomain borders marked by the thick lines in Figure 3, are denoted by $\mathcal{H}_{H}$ for the coarse mesh and by $\mathcal{H}_{h}$ for the fine mesh. The hybrid flux vector is used to enforce continuity on functions in $\hat{X}_{H}$ and $\hat{X}_{h}$, in particular, as 'glue' to 'connect' the adjacent nodes, lines or faces so that the noncomforming velocities in different subdomains can be continuous 


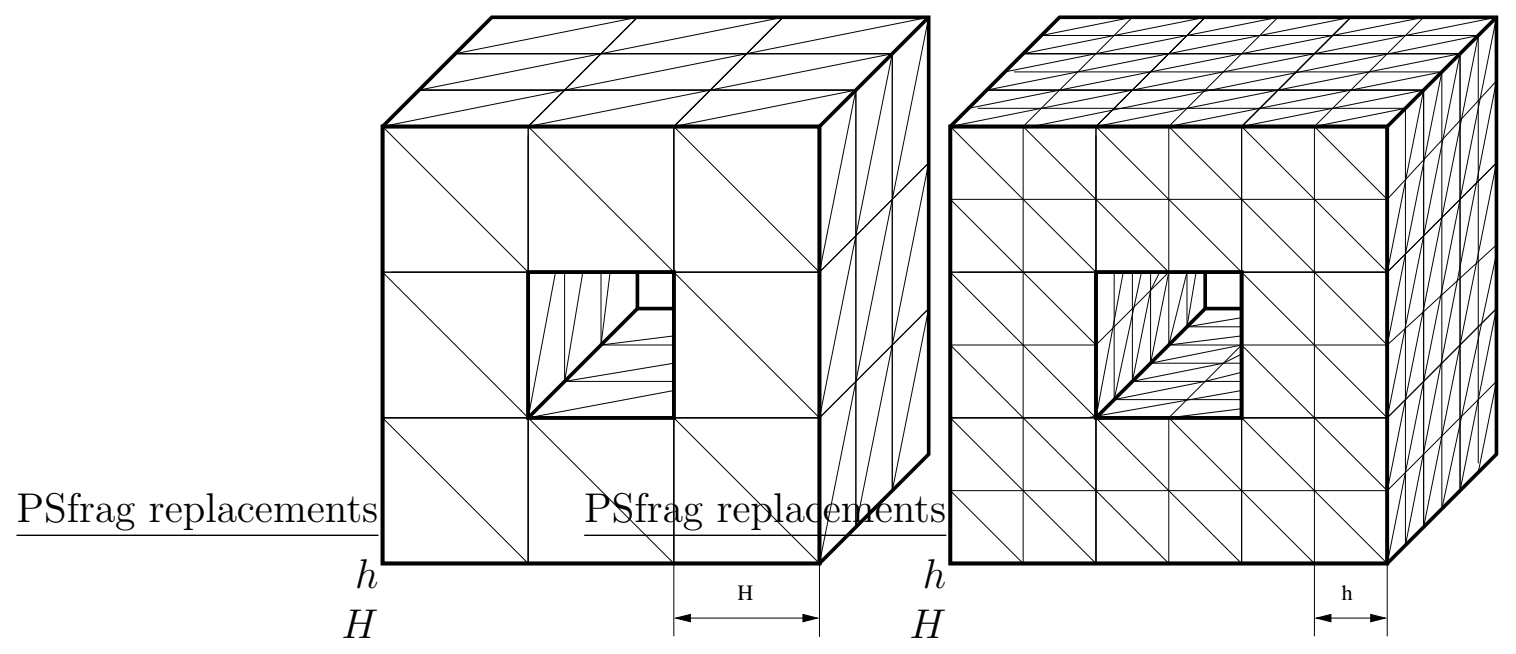

Figure 2: The coarse $H$ mesh $\left(\mathcal{T}_{H}\right.$, left $)$ and the fine $h$ mesh $\left(\mathcal{T}_{h}\right.$, right) before the domain decomposition $\left(R=\frac{H}{h}=2\right)$.

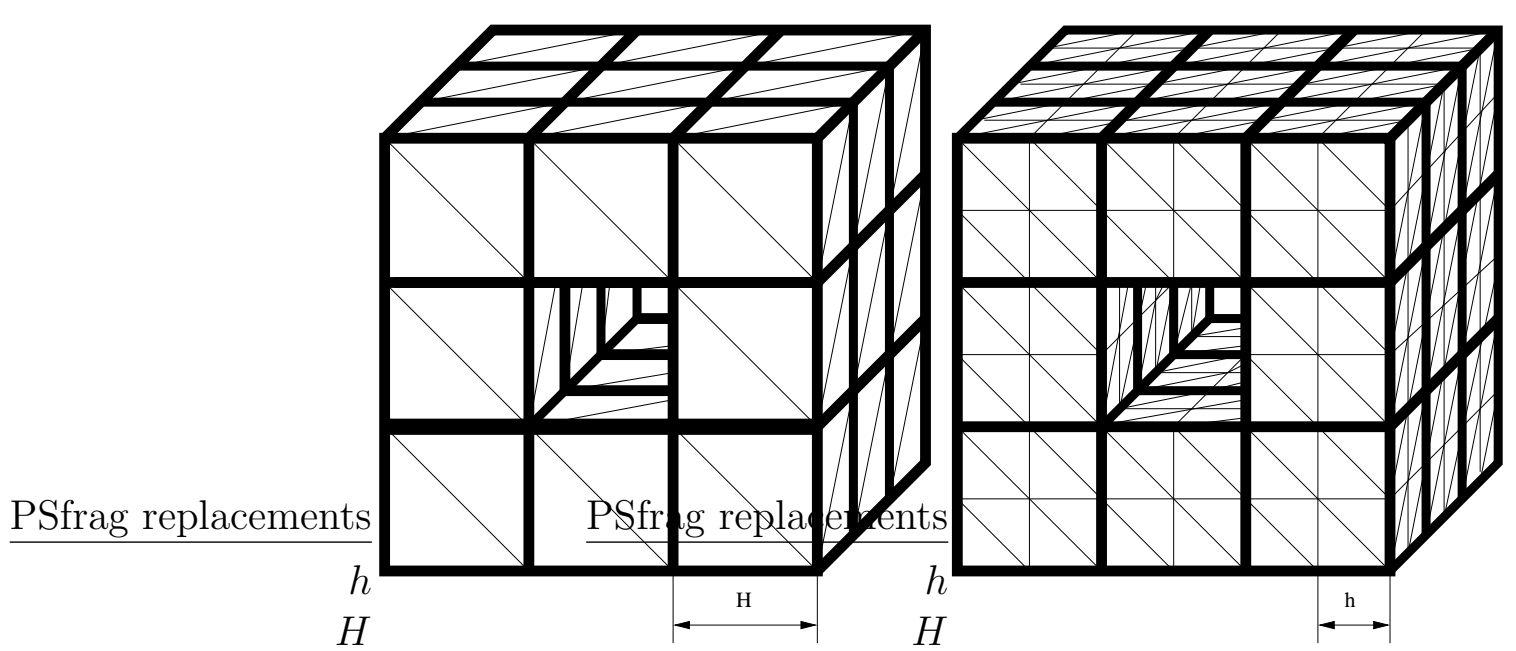

Figure 3: The coarse $H$ mesh $\left(\mathcal{T}_{H}\right.$, left $)$ and the fine $h$ mesh $\left(\mathcal{T}_{h}\right.$, right $)$ after the domain decomposition $\left(R=\frac{H}{h}=2\right)$. The thick lines represent subdomain borders.

over the whole domain. In this paper, the superscript ${ }^{(k)}$ represents a parameter identifying the subdomain $\Omega^{(k)}$.

Finite element coarse mesh solution $\left(u_{1 H}, u_{2 H}, u_{3 H}, p_{H}\right) \in X_{H} \times \mathcal{Q}_{H}$ and fine mesh solution $\left(u_{1 h}, u_{2 h}, u_{3 h}, p_{h}\right) \in X_{h} \times \mathcal{Q}_{h}$ satisfy the $\mathrm{N}-\mathrm{S}$ weak formulations in matrix form

$$
\begin{aligned}
\left(A_{H}+C_{H}\right) \bar{u}_{i H}+B_{i H}^{T} \bar{p}_{H} & =\bar{f}_{i H}, \quad i=1,2,3, \\
\sum_{i=1}^{3} B_{i H} \bar{u}_{i H} & =0 \\
\left(A_{h}+C_{h}\right) \bar{u}_{i h}+B_{i h}^{T} \bar{p}_{h} & =\bar{f}_{i h}, \quad i=1,2,3, \\
\sum_{i=1}^{3} B_{i h} \bar{u}_{i h} & =0
\end{aligned}
$$


where matrices $A_{H}, C_{H}$, and $B_{i H}$ are obtained from the coarse mesh function space $X_{H}$ and matrices $A_{h}, C_{h}$, and $B_{i h}$ are obtained from the fine one $X_{h}$. The average velocity becomes $s_{H}=\bar{\ell}_{H}^{0} \bar{u}_{3 H}=1_{H}^{T} M_{H} \bar{u}_{3 H}$ for the coarse mesh, and and $s_{h}=\bar{\ell}_{h}^{0^{T}} \bar{u}_{3 h}=1_{h}^{T} M_{h} \bar{u}_{3 h}$ for the fine mesh. Here, $M_{H}$ and $M_{h}$ denote the mass matrices on the coarse and fine mesh space, respectively.

\section{Augmented Lagrangian Formulation}

A novel approach in the current bound method is to exploit the coarse mesh linearized N-S equations to construct a fine mesh augmented Lagrangian, which also includes a quadratic 'energy' reformation of the desired output, and which contains the inter-subdmain continuity requirements as well. From the quadratic-linear duality theory of the Lagrangian, the bounding properties can be easily proved. This formulation is different from that used for the "general asymptotic finite element bound method" described in detail in [11], since a Lagrangian formulation is used. For a more detailed description of previous a posteriori finite element method related to error estimation for the $\mathrm{N}-\mathrm{S}$ equations, the reader is referred to $[15,22]$.

\subsection{The construction of the Lagrangian}

To construct the proper energy equality for the augmented Lagrangian, let $\mathbf{e}_{h}=\mathbf{u}_{h}-\mathbf{u}_{H}$, $\varepsilon_{h}=p_{h}-p_{H}$. Note that $\mathbf{e}_{h} \in X_{h}$ and $\varepsilon_{h} \in \mathcal{Q}_{h}$ are associated with the errors for the velocity and pressure respectively.

\subsubsection{General residuals}

The coarse mesh solution, $\left(\mathbf{u}_{H}=\left(u_{1 H}, u_{2 H}, u_{3 H}\right), p_{H}\right) \in X_{H} \times \mathcal{Q}_{H}$, can be used to define the primal residuals of the $\mathrm{N}-\mathrm{S}$ equations on each subdomain, $\Omega^{(k)}$ with $k=1, \cdots, N_{k}$, for the 'broken' space on both meshes:

$$
\begin{aligned}
\bar{\ell}_{i \delta}^{(k)} & \equiv \bar{f}_{i \delta}^{(k)}-\left[A_{\delta}^{(k)}+D_{\delta}^{(k)}\right] \bar{I}^{\delta}\left(u_{i H}^{(k)}\right)-B_{i \delta}^{(k)^{T}} \bar{I}^{\delta}\left(p_{H}^{(k)}\right), \quad(i=1,2,3), \\
\bar{b}_{\delta}^{(k)} & \equiv-\sum_{i=1}^{3} B_{i \delta}^{(k)} \bar{I}^{\delta}\left(u_{i H}^{(k)}\right)
\end{aligned}
$$

where $\bar{I}^{\delta}(\cdot)$ represents the vector of coefficients of a function interpolated onto a function space parameterized by $\delta$.

For $N_{k}$ subdomains, $u_{i H}=\left\{u_{i H}^{(1)}, \cdots, u_{i H}^{\left(N_{k}\right)}\right\}$ with $i=1,2,3$ and $p_{H}=\left\{p_{H}^{(1)}, \cdots, p_{H}^{\left(N_{k}\right)}\right\}$. $A_{\delta}^{(k)}, D_{\delta}^{(k)}$ and $B_{i \delta}^{(k)}$ are the local matrices associated with $A_{\delta}, D_{\delta}$ and $B_{i \delta}$ respectively, on each subdomain, $\Omega^{(k)}$.

The energy equality on the 'broken' fine $h$-mesh can be written as follows:

$$
\begin{aligned}
& \sum_{k=1}^{N_{k}} \sum_{i=1}^{3}\left[\bar{e}_{i h}^{(k)} A_{h}^{(k)} \bar{e}_{i h}^{(k)}+2 \bar{\varepsilon}_{h}^{(k)^{T}} B_{i h}^{(k)} \bar{e}_{i h}^{(k)}-\bar{e}_{i h}^{(k)^{T}} \bar{\ell}_{i h}^{(k)}-\bar{\varepsilon}_{h}^{(k)^{T}} \bar{b}_{h}^{(k)}\right] \\
& =\sum_{k=1}^{N_{k}} \sum_{i=1}^{3}\left[\bar{e}_{i h}^{(k)^{T}} A_{h}^{(k)} \bar{e}_{i h}^{(k)}+2 \bar{e}_{i h}^{(k)^{T}} B_{i h}^{(k)^{T}} \bar{\varepsilon}_{h}^{(k)}+\bar{e}_{i h}^{(k)^{T}}\left(A_{h}^{(k)}+D_{h}^{(k)}\right) \bar{I}^{h}\left(u_{i H}^{(k)}\right)+\bar{e}_{i h}^{(k)^{T}} B_{i h}^{(k)}{ }^{T} \bar{I}^{h}\left(p_{H}^{(k)}\right)\right.
\end{aligned}
$$




$$
\left.-\bar{e}_{i h}^{(k)^{T}} \bar{f}_{i h}^{(k)}+\bar{\varepsilon}_{h}^{(k)^{T}} B_{i h}^{(k)} \bar{I}^{h}\left(u_{i H}^{(k)}\right)\right] .
$$

If the mesh size $h=H$, the coarse mesh matrices and solutions will be the same as the fine, $\left(\mathbf{e}_{h}^{(k)}, \varepsilon_{h}^{(k)}\right)=\left(e_{1 h}^{(k)}, e_{2 h}^{(k)}, e_{3 h}^{(k)}, \varepsilon_{h}^{(k)}\right)=0$, and the energy equality (19) will vanish.

\subsubsection{Energy reformation of outputs}

Thus, the following trivial minimization statement is introduced with the new energy equality and the linear output of interest of the Navier-Stokes equations:

$$
\begin{gathered}
\pm s_{h}\left(\mathbf{v}_{h}, q_{h}\right) \\
=\inf _{\mathbf{v}_{h} \in \hat{X}_{h}} \sup _{q_{h} \in \mathcal{Q}_{h}} \sum_{k=1}^{N_{k}} \sum_{i=1}^{3}\left\{\kappa\left[\bar{v}_{i h}^{(k)} A_{h}^{(k)} \bar{v}_{i h}^{(k)}+2 \bar{q}_{h}^{(k)^{T}} B_{i h}^{(k)} \bar{v}_{i h}^{(k)}-\bar{\ell}_{i h}^{(k)^{T}} \bar{v}_{i h}^{(k)}-\bar{b}^{(k)^{T}} \bar{q}^{(k)}\right] \pm \bar{\ell}_{h}^{0^{T}}\left(\bar{I}^{h}\left(u_{i H}^{(k)}\right)+\bar{v}_{i h}^{(k)}\right)\right. \\
=\inf _{\mathbf{v}_{h} \in \hat{X}_{h}} \sup _{q_{h} \in \mathcal{Q}_{h}} \sum_{k=1}^{N_{k}} \sum_{i=1}^{3}\left\{\kappa \left[\bar{v}_{i h}^{(k)^{T}} A_{h}^{(k)} \bar{v}_{i h}^{(k)}+2 \bar{q}_{h}^{(k)^{T}} B_{i h}^{(k)} \bar{v}_{i h}^{(k)}+\bar{v}_{i h}^{(k)^{T}} B_{i h}^{(k)} \bar{I}^{h}\left(p_{H}^{(k)}\right)\right.\right. \\
\left.+\bar{v}_{i h}^{(k)^{T}}\left(A_{h}^{(k)}+D_{h}^{(k)}\right) \bar{I}^{h}\left(u_{i H}^{(k)}\right)-\bar{v}_{i h}^{(k)} \bar{f}_{i h}^{(k)}+\bar{q}_{h}^{(k)} B_{i h}^{(k)} \bar{I}^{h}\left(u_{i H}^{(k)}\right)\right] \\
\left. \pm \bar{\ell}_{h}^{0^{T}}\left(\bar{I}^{h}\left(u_{i H}^{(k)}\right)+\bar{v}_{i h}^{(k)}\right)\right\}
\end{gathered}
$$

where $q_{h}$ refers to the variables associated with the pressure error, $\mathbf{v}_{h}=\left(v_{1 h}, v_{2 h}, v_{3 h}\right)$ associated with the velocity error, and $\kappa$ is a non-negative parameter that will be used to optimize the computed bounds. Note again that the overbar,$\cdot \overline{,}$, represents the vector of coefficients that defines the respective function.

\subsubsection{Linearized $\mathrm{N}-\mathrm{S}$ equations and inter-subdomain constraints}

Now the set of functions $\mathcal{S} \subset \hat{X}_{h} \times \mathcal{Q}_{h}$ is introduced for the construction of the linearized Navier-Stokes equations constrained by adjoints, $\left(\mathbf{w}_{h}^{(k)}, r_{h}^{(k)}\right)=\left(\omega_{1 h}^{(k)}, \omega_{2 h}^{(k)}, \omega_{3 h}^{(k)}, r^{(k)}\right)$, from equations (11) and (12). The high order convection term, which is very small, is omitted in this procedure so that no non-linear convection term will appear in the new augmented Lagrangian. $H_{\delta}^{(k)}$ is defined as the Boolean matrix which extracts the signed $( \pm)$ restriction of a subdomain solution $\mathbf{v}_{h}^{(k)}=\left(v_{1 h}^{(k)}, v_{2 h}^{(k)}, v_{3 h}^{(k)}\right)$ to the subdomain interface boundary. Lagrange multiplier $\mu_{h}=\left(\mu_{1 h}, \mu_{2 h}, \mu_{3 h}\right) \in \mathcal{H}_{h}$ reflect the hybrid flux. The constraint $\bar{\mu}_{i h}^{T} H_{h}^{(k)} \bar{v}_{i h}^{(k)}=0, \forall \mu_{h} \in \mathcal{H}_{h}$, enforces velocity continuity and the homogeneous essential conditions. For non-homogeneous boundary conditions, a vector of coefficients representing the function on the boundary should be subtracted on the left-hand side of this equality.

with $i=1,2,3$.

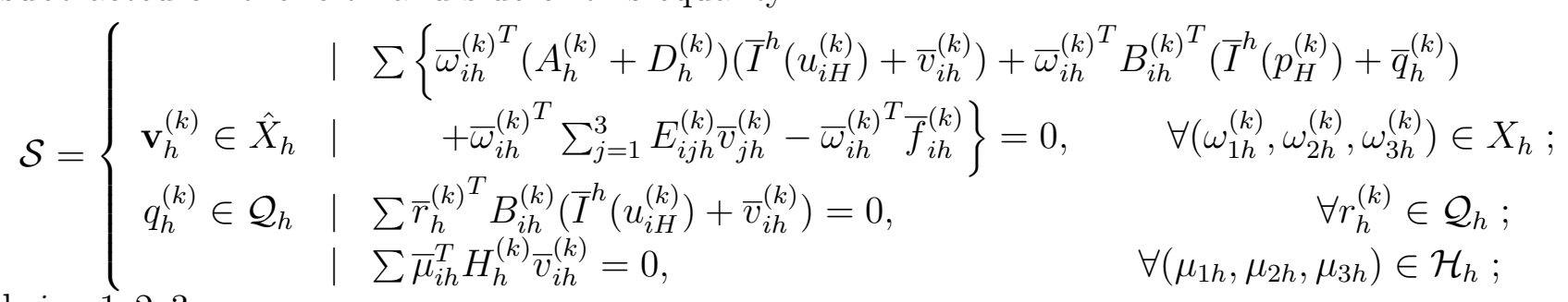




\subsubsection{Quadratic-linear Lagrangian}

The constrained minimization problem suggests the formation of the quadratic-linear Lagrangian $\mathcal{L}: \hat{X}_{h} \times \mathcal{Q}_{h} \times X_{h} \times \mathcal{Q}_{h} \times \mathcal{H}_{h} \rightarrow \mathbf{R}$

$$
\begin{aligned}
\mathcal{L}^{ \pm}\left(\mathbf{v}_{h}, q_{h}, \mathbf{w}_{h}, r_{h}, \mu_{h}\right) \\
=\sum_{i=1}^{3} \sum_{k=1}^{N_{k}}\left\{\left[\kappa \left(\bar{v}_{i h}^{(k)^{T}} A_{h}^{(k)} \bar{v}_{i h}^{(k)}+2 \bar{q}_{h}^{(k)^{T}} B_{i h}^{(k)} \bar{v}_{i h}^{(k)}+\bar{v}_{i h}^{(k)^{T}} B_{i h}^{(k)}{ }^{T} \bar{I}^{h}\left(p_{H}^{(k)}\right)-\bar{v}_{i h}^{(k)^{T}} \bar{f}_{i h}^{(k)}\right.\right.\right. \\
\left.\left.\quad+\bar{v}_{i h}^{(k)}\left(A_{h}^{(k)}+D_{h}^{(k)}\right) \bar{I}^{h}\left(u_{i H}^{(k)}\right)+\bar{q}_{h}^{(k)}{ }^{T} B_{i h}^{(k)} \bar{I}^{h}\left(u_{i H}^{(k)}\right)\right) \pm \bar{\ell}_{h}^{0 T}\left(\bar{I}^{h}\left(u_{i H}^{(k)}\right)+\bar{v}_{i h}^{(k)}\right)\right] \\
+\left[\bar{\omega}_{i h}^{(k)}\right)^{T}\left(A_{h}^{(k)}+D_{h}^{(k)}\right)\left(\bar{I}^{h}\left(u_{i H}^{(k)}\right)+\bar{v}_{i h}^{(k)}\right)+\bar{\omega}_{i h}^{(k)^{T}} B_{i h}^{(k)^{T}}\left(\bar{I}^{h}\left(p_{H}^{(k)}\right)+\bar{q}_{h}^{(k)}\right)-\bar{\omega}_{i h}^{(k)^{T}} \bar{f}_{i h}^{(k)} \\
\left.\left.+\bar{\omega}_{i h}^{(k)} \sum_{j=1}^{3} E_{i j h}^{(k)} \bar{v}_{j h}^{(k)}+\bar{r}_{h}^{(k)^{T}} B_{i h}^{(k)}\left(\bar{I}^{h}\left(u_{i H}^{(k)}\right)+\bar{v}_{i h}^{(k)}\right)\right]+\bar{\mu}_{i h}^{T} H_{h}^{(k)} \bar{v}_{i h}^{(k)}\right\}
\end{aligned}
$$

where the first two lines represent the quadratic 'energy' reformation of the desired output, the third and fourth lines represent the linearized Navier-Stokes problem constrained by the Lagrange multiplies $\mathbf{w}_{h}$ and $r_{h}$, and the last term represents the velocity continuity requirements for the inter-subdomains. The Lagrangian (21) is quadratic in its argument $\mathbf{v}$ and linear in its $\operatorname{arguments} q_{h}, \mathbf{w}_{h}, r_{h}$ and $\mu_{h}$ and its saddle point $\left(\hat{\mathbf{e}}_{h}, \varepsilon_{h}^{ \pm}, \psi_{h}^{ \pm}, \lambda_{h}^{ \pm}, \mathbf{t}_{h}^{ \pm}\right)$is obtained by

$$
\begin{aligned}
\hat{e}_{h} & =\underset{\mathbf{v}_{h} \in \hat{X}_{h}}{\arg \min }\left[\sup _{q_{h} \in \mathcal{Q}_{h}, \mathbf{w}_{h} \in X_{h}, r_{h} \in \mathcal{Q}_{h}, \mu_{h} \in \mathbf{E}_{h}} \mathcal{L}^{ \pm}\left(\mathbf{v}_{h}, q_{h}, \mathbf{w}_{h}, r_{h}, \mu_{h}\right)\right], \\
\left(\varepsilon_{h}^{ \pm}, \psi_{h}^{ \pm}, \lambda_{h}^{ \pm}, \mathbf{t}_{h}^{ \pm}\right) & =\underset{q_{h} \in \mathcal{Q}_{h}, \mathbf{w}_{h} \in X_{h}, r_{h} \in \mathcal{Q}_{h}, \mu_{h} \in \mathcal{H}_{h}}{\arg }\left[\underset{\mathbf{v} \in \hat{X}_{h}}{\max } \mathcal{L}^{ \pm}\left(\mathbf{v}_{h}, q_{h}, \mathbf{w}_{h}, r_{h}, \mu_{h}\right)\right] .
\end{aligned}
$$

\subsection{Proofing of the bounding properties}

From the quadratic-linear duality theory, it follows that

$$
\begin{aligned}
\pm s_{h} & =\sup _{q_{h} \in \mathcal{Q}_{h}, \mathbf{w}_{h} \in X_{h}, r_{h} \in \mathcal{Q}_{h}, \mu \in \mathcal{H}_{h}} \inf _{\mathbf{v}_{h} \in \hat{X}_{h}} \mathcal{L}^{ \pm}\left(\mathbf{v}_{h}, q_{h}, \mathbf{w}_{h}, r_{h}, \mu_{h}\right) \\
& \geq \inf _{\mathbf{v}_{h} \in \hat{X}_{h}} \mathcal{L}^{ \pm}\left(\mathbf{v}_{h}, \check{q}_{h}^{ \pm}, \check{\mathbf{w}}_{h}^{ \pm}, \check{r}_{h}^{ \pm}, \check{\mu}_{h}^{ \pm}\right), \quad \forall \check{q}_{h}^{ \pm} \in \mathcal{Q}_{h}, \forall \check{\mathbf{w}}_{h}^{ \pm} \in X_{h}, \forall \check{r}_{h}^{ \pm} \in \mathcal{Q}_{h}, \forall \check{\mu}_{h}^{ \pm} \in \mathcal{H}_{h},
\end{aligned}
$$

with equality for $\left(\check{q}_{h}^{ \pm}, \check{\mathbf{w}}_{h}^{ \pm}, \check{r}_{h}^{ \pm}, \check{\mu}_{h}^{ \pm}\right)=\left(\varepsilon_{h}^{ \pm}, \psi_{h}^{ \pm}, \lambda_{h}^{ \pm}, \mathbf{t}_{h}^{ \pm}\right)$.

Upper and lower bounds for the output $s_{h}, s_{U B}$, and $s_{L B}$, respectively, are now readily constructed as

$$
s_{L B} \equiv \inf _{\mathbf{v} \in \hat{X}_{h}} \mathcal{L}^{+}\left(\mathbf{v}, \check{q}_{h}^{+}, \check{\mathbf{w}}_{h}^{+}, \check{r}_{h}^{+}, \check{\mu}_{h}^{+}\right) \leq s_{h} \leq-\inf _{\mathbf{v} \in \hat{X}_{h}} \mathcal{L}^{-}\left(\mathbf{v}, \check{q}_{h}^{-}, \check{\mathbf{w}}_{h}^{-}, \check{r}_{h}^{-}, \check{\mu}_{h}^{-}\right) \equiv s_{U B}
$$

where the superscripts, $(\star)^{+}$and $(\star)^{-}$, indicate the upper and lower bounds of the arguments $(\star)$, respectively. Note that equation (25) holds for any functions $\left(\check{q}_{h}^{ \pm}, \check{\mathbf{w}}_{h}^{ \pm}, \check{r}_{h}^{ \pm}, \check{\mu}_{h}^{ \pm}\right) \in\left(\mathcal{Q}_{h} \times X_{h} \times\right.$ $\left.\mathcal{Q}_{h} \times \mathcal{H}_{h}\right)$. 


\section{Bound calculations}

Starting from the Lagrangian, the algebraic formulation of the bound procedure for outputs of the Navier-Stokes problem will be exposed.

Equations (21), (24) and (25) suggest that the bound procedure for the Navier-Stokes problem require the calculations of the field solution $\left(\mathbf{u}_{H}, p_{H}\right)$, the three Lagrange multiplier candidates, adjoint $\left(\psi_{H}^{ \pm}, \lambda_{H}^{ \pm}\right)$and hybrid flux $\mathbf{t}_{H}^{ \pm}$, on the global coarse mesh $\mathcal{T}_{H}$, and the calculations of the restructured errors $\hat{\mathbf{e}}_{h}^{ \pm}$and $\hat{\varepsilon}_{h}^{ \pm}$, on the decoupled fine mesh $\mathcal{T}_{h}$. These parameters can be inexpensively calculated by solving a global, coarse field solution (primal) problem, a coarse adjoint (dual) problem, a coarse hybrid flux problem and $N_{k}$ subdomain fine mesh problems, which are simplified from the stationarity conditions of the augmented Lagrangian (21).

The stationarity conditions are: find $\mathbf{e}_{H}^{ \pm} \in \hat{X}_{H}, \varepsilon_{H}^{ \pm} \in \mathcal{Q}_{H}, \psi_{H}^{ \pm} \in X_{H}, \lambda_{H}^{ \pm} \in \mathcal{Q}_{H}$, and $\mathbf{t}_{H}^{ \pm} \in \mathcal{H}_{H}$ such that the vector coefficients of these functions satisfy:

$$
\begin{aligned}
& \kappa\left\{2 A_{H}^{(k)} \bar{e}_{i H}^{(k)^{ \pm}}+2 B_{i H}^{(k)^{T}} \bar{\varepsilon}_{H}^{(k)^{ \pm}}+\left[A_{H}^{(k)}+D_{H}^{(k)}\right] \bar{u}_{i H}^{(k)}+B_{i H}^{(k)^{T}} \bar{p}_{H}^{(k)}-\bar{f}_{i H}^{(k)}\right\}+\left\{\left[A_{H}^{(k)}+D_{H}^{(k)^{T}}\right] \bar{\psi}_{i H}^{(k)^{ \pm}}\right. \\
& \left.+B_{i H}^{(k)} \bar{\lambda}_{H}^{(k)^{ \pm}}+\sum_{j=1}^{3} E_{j i H}^{(k)} \bar{\psi}_{j H}^{(k)^{ \pm}} \pm \bar{\ell}_{i H}^{0}\right\}+H_{H}^{(k)^{T}} \bar{t}_{i H}^{ \pm}=0, \\
& \kappa\left\{2 B_{i H}^{(k)} \bar{e}_{i H}^{(k)^{ \pm}}+B_{i H}^{(k)} \bar{u}_{i H}^{(k)}\right\}+B_{i H}^{(k)} \bar{\psi}_{i H}^{(k)^{ \pm}}=0, \\
& H_{H}^{(k)} \bar{e}_{i H}^{(k)^{ \pm}}=0, \\
& {\left[A_{H}^{(k)}+D_{H}^{(k)}\right]\left[\bar{u}_{i H}^{(k)}+\bar{e}_{i H}^{(k) \pm}\right]+B_{i H}^{(k)^{T}}\left[\bar{p}_{H}^{(k)}+\bar{\varepsilon}_{H}^{(k)^{ \pm}}\right]+\sum_{j=1}^{3} E_{j i H}^{(k)} \bar{e}_{j H}^{(k)} \pm=\bar{f}_{i H}^{(k)},} \\
& B_{i H}^{(k)} \bar{u}_{i H}^{(k)}+B_{i H}^{(k)} \bar{e}_{i H}^{(k)^{ \pm}}=0 .
\end{aligned}
$$

Equation (28) forces $\mathbf{e}_{H}^{ \pm} \in X_{H}$, and $\left(\mathbf{e}_{H}^{ \pm}, \varepsilon_{H}^{ \pm}\right)=0$ has to be satisfied for $\left(\mathbf{e}_{H}^{ \pm}, \varepsilon_{H}^{ \pm}\right) \in X_{H} \times \mathcal{Q}_{H}$.

\subsection{The primal problem}

By introducing $\left(\mathbf{e}_{H}^{ \pm}, \varepsilon_{H}^{ \pm}\right)=0$, equations (29) and (30) lead to the primal problem which is relatively standard: find the global field solution $\left(\mathbf{u}_{H}, p_{H}\right)$ that satisfies the original non-linear Navier-Stokes problem in matrix form, (7) and (8), on the coarse $H$-mesh:

$$
\begin{aligned}
\left(A_{H}+C_{H}\right) \bar{u}_{i H}+B_{i H}^{T} \bar{p}_{H} & =\bar{f}_{i H}, \quad i=1,2,3 \\
\sum_{i=1}^{3} B_{i H} \bar{u}_{i H} & =0
\end{aligned}
$$

where the matrix $C_{H}$ should be determined by the field solution $u_{i H}$. Note that the convection term $C_{H} u_{i H}$ is nonlinear. Newton's method, a quadratically converging process described in section 2.2, is suitable for the iterative calculation of these nonlinear Navier-Stokes equations. A Generalized Minimal Residual (GMRES) solver [19] with ILU(0) preconditioner is used for solving the linearized non-symmetric algebraic systems (11) and (12) within each Newton iteration step. 


\subsection{The dual problem}

Forcing the subdomain solution $\mathbf{u}_{H}^{(k)}$ and adjoint vector $\psi_{H}^{(k)^{ \pm}}$to be continuous in the whole domain $\Omega$ can eliminate the hybrid flux term $H_{H}^{(k)}{ }^{T} \bar{t}_{i H}^{ \pm}$in equation (26), because no intersubdomain jumps exist for $\mathbf{u}_{H}$ and $\psi_{H}^{ \pm}$in the conforming space $X_{H}$.

By setting $\psi_{i H}^{ \pm}= \pm \psi_{i H}$ and $\lambda_{H}^{ \pm}= \pm \lambda_{H}$, and by evoking equations (31) and (32), equations (26) and (27) simplify as the dual problem: find the adjoint solution $\left(\psi_{1 H}, \psi_{2 H}, \psi_{3 H}, \lambda_{H}\right) \in$ $X_{H} \times \mathcal{Q}_{H}$, such that

$$
\begin{aligned}
\left(A_{H}+D_{H}^{T}\right) \bar{\psi}_{i H}+\sum_{j=1}^{3} E_{j i H}^{T} \bar{\psi}_{j H}+B_{i H}^{T} \bar{\lambda}_{H} & =-\bar{\ell}^{0} \\
\sum_{i=1}^{3} B_{i H} \bar{\psi}_{i H} & =0
\end{aligned}
$$

The dual problem is linear, so no Newton iteration is required for solving (33) and (34). GMRES solver with ILU(0) preconditioner is used to solve the underlying nonsymmetric algebraic system.

\subsection{The hybrid flux problem}

Let $\mathbf{e}_{H}^{ \pm(k)}=\mathbf{e}_{H}^{p r(k)} \pm \frac{1}{\kappa} \mathbf{e}_{H}^{d u}{ }^{(k)}, \varepsilon_{H}^{ \pm}{ }^{(k)}=\varepsilon_{H}^{p r(k)} \pm \frac{1}{\kappa} \varepsilon_{H}^{d u}{ }^{(k)}$, and $\mathbf{t}_{H}^{ \pm}=\kappa \mathbf{t}_{H}^{p r} \pm \mathbf{t}_{H}^{d u}$. Substituting these parameters and $\left(\psi_{H}^{ \pm}{ }^{(k)}, \lambda_{H}^{ \pm}{ }^{(k)}\right)= \pm\left(\psi_{i H}^{(k)}, \lambda_{H}^{(k)}\right)$ into the stationarity conditions (26), (27) and (28) leads to the following two hybrid flux problems:

- The primal hybrid flux problem: find $\left(t_{1 H}^{p r}, t_{2 H}^{p r}, t_{3 H}^{p r}\right) \in \mathbf{E}_{H}$ such that

$$
\begin{aligned}
& 2 A_{H}^{(k)} \bar{e}_{i H}^{p r(k)}+2 B_{i H}^{(k)^{T}} \bar{\varepsilon}_{H}^{p r(k)}+H_{H}^{(k)} \bar{t}_{i H}^{p r}+\left[A_{H}^{(k)}+D_{H}^{(k)}\right] \bar{u}_{i H}^{(k)}+B_{i H}^{(k)^{T}} \bar{p}_{H}^{(k)}-\bar{f}_{i H}^{(k)}=0 \\
& 2 \sum_{i=1}^{3} B_{i H}^{(k)} \bar{e}_{i H}^{p r}{ }^{(k)}=-\sum_{i=1}^{3} B_{i H}^{(k)} \bar{u}_{i H}^{(k)} \\
& \sum_{k=1}^{N_{k}} H_{H}^{(k)} \bar{e}_{i H}^{p r(k)}=0
\end{aligned}
$$

- The dual hybrid flux problem: find $\left(t_{1 H}^{d u}, t_{2 H}^{d u}, t_{3 H}^{d u}\right) \in \mathbf{E}_{H}$ such that

$$
\begin{aligned}
& 2 A_{H}^{(k)} \bar{e}_{i H}^{d u}(k)+2 B_{i H}^{(k)^{T}} \bar{\varepsilon}_{H}^{d u}(k)+H_{H}^{(k)^{T}} \bar{t}_{i H}^{d u}+\bar{\ell}_{i H}^{0}+\left[\left(A_{H}^{(k)}+D_{H}^{(k)^{T}}\right] \bar{\psi}_{i H}^{(k)}+B_{i H}^{(k)} \bar{\lambda}_{H}^{(k)}\right. \\
& +\sum_{j=1}^{3} E_{j i H}^{(k)} \bar{\psi}_{j H}^{(k)}=0 \\
& 2 \sum_{i=1}^{3} B_{i H}^{(k)} \bar{e}_{i H}^{d u}{ }^{(k)}=-\sum_{i=1}^{3} B_{i H}^{(k)} \bar{\psi}_{i H}^{(k)} \\
& \sum_{k=1}^{N_{k}} H_{H}^{(k)} \bar{e}_{i H}^{d u}{ }^{(k)}=0 .
\end{aligned}
$$




\subsubsection{The hybrid flux associated with the primal problem}

The primal hybrid flux problem can be further expressed in the standard FETI interface formulations for each hybrid flux component $t_{i H}^{p r}$ in the $x_{i}$ direction $(i=1,2,3)[3]$.

Because the Crouzeix-Raviart pressure space is discontinuous, mass conservation can always be satisfied in each tetrahedron and subdomain, if conservation is also satisfied in the global domain. That is, if $\sum_{i=1}^{3} B_{H} \bar{u}_{i H}=0$, then $\sum_{i=1}^{3} B_{H}^{(k)} \bar{u}_{i H}^{(k)}=0, \quad \forall k: 1 \leq k \leq N_{k}$.

With $e_{1 H}^{p r}{ }^{(k)}=e_{2 H}^{p^{(k)}}=e_{3 H}^{p r}{ }^{(k)}=0$ and $\varepsilon_{H}^{p r(k)}=0$, equation (35) can be simplified as:

$$
2 A_{H}^{(k)} \bar{e}_{i H}^{p r(k)}+\left[A_{H}^{(k)} \bar{u}_{i H}^{(k)}+B_{i H}^{(k)}{ }^{T} \bar{p}_{H}^{(k)}-\bar{f}_{i H}^{(k)}\right]+H_{H}^{(k)^{T}} \bar{t}_{i H}^{p r}=0, \quad(i=1,2,3),
$$

where $A_{H}^{(k)} \bar{e}_{i H}^{p r}{ }^{(k)}$ is an artificial term, which is equal to zero.

Now all three components of the hybrid flux vector are decoupled, and the above hybrid flux equation can be solved inexpensively. Let the primal residual $\bar{q}_{i H}^{p r(k)}=\bar{f}_{i H}^{(k)}-\left[A_{H}^{(k)} \bar{u}_{i H}^{(k)}+B_{i H}^{(k)}{ }^{T} \bar{p}_{H}^{(k)}\right]$, $K_{H}^{(k)}=2 A_{H}^{(k)}$, and $R_{H}^{(k)}$ be the null space of $A_{H}^{(k)}$, namely, $R_{H}^{(k)} \perp A_{H}^{(k)}$. Then equation (41) leads to

$$
K_{H}^{(k)} \bar{e}_{i H}^{p r(k)}+H_{H}^{(k)}{ }^{T} \bar{t}_{i H}^{p r}=\bar{q}_{i H}^{p r(k)}
$$

By defining $K_{H}^{(k)^{+}}$as the inverse matrix of $K_{H}^{(k)}$, equation (42) is equivalent to

$$
\begin{aligned}
\bar{e}_{i H}^{p r(k)} & =K_{H}^{(k)^{+}}\left[\bar{q}_{i H}^{p r(k)}-H_{H}^{(k)^{T}} \bar{t}_{i H}^{p r}\right]+R^{(k)} \bar{\alpha}_{i H}^{p r}{ }^{(k)}, \\
0 & =R^{(k)^{T}}\left(\bar{q}_{i H}^{p r(k)}-H_{H}^{(k)^{T}} \bar{t}_{i H}^{p r}\right),
\end{aligned}
$$

where $\bar{\alpha}_{i H}^{p r}{ }^{(k)}$ denotes the set of amplitudes that specifies the shifting of the subdomain $\Omega^{(k)}$ for the residual of the primal problem (31).

The substitution of (43) into (37) to eliminate $\bar{e}_{i H}^{p r(k)}$ leads to the following dual interface problem: find $t_{i H}^{p r}$ such that

$$
\begin{aligned}
\sum_{k=1}^{N_{k}} H_{H}^{(k)} K_{H}^{(k)+}\left[\bar{q}_{i H}^{p r}{ }^{(k)}-H_{H}^{(k)^{T}} \bar{t}_{i H}^{p r}\right]+\sum_{k=1}^{N_{k}} H_{H}^{(k)} R^{(k)} \bar{\alpha}_{i H}^{p r}{ }^{(k)} & =0, \\
R^{(k)^{T}}\left(\bar{q}_{i H}^{p r(k)}-H_{H}^{(k)^{T}} \bar{t}_{i H}^{p r}\right) & =0 .
\end{aligned}
$$

The dual interface problem (45) and (46) for $t_{i H}^{p r}$ can be written in the standard FETI interface formulations:

where

$$
\left[\begin{array}{cc}
F_{H} & -G_{H} \\
-G_{H}^{T} & 0
\end{array}\right]\left[\begin{array}{c}
\bar{t}_{i H}^{p r} \\
\bar{\alpha}_{i H}^{p r}
\end{array}\right]=\left[\begin{array}{c}
\bar{d}_{i H}^{p r} \\
-\bar{g}_{i H}^{p r}
\end{array}\right], i=\{1,2,3\}
$$


The interface problem (47) is applied to each component of velocity $(i=1,2,3)$, which can be solved separately by a preconditioned conjugate projected gradient (PCPG) algorithm. A projection operator is exploited to handle the self-equilibrium constraints. The FETI method iterates on $\bar{t}_{i H}^{p r}$, given an initial $\bar{t}_{i H}^{p r} 0$ which satisfies the constraint $G_{H}^{T} \bar{t}_{i H}^{p r}{ }^{0}=\bar{g}_{H}^{p r}$. Subsequent increments of the inter-subdomain connectivity satisfy:

$$
\begin{aligned}
P_{H} F_{H} \Delta \bar{t}_{i H}^{p r} & =P_{H}\left(\bar{d}_{i H}^{p r}-F_{H} \bar{t}_{i H}^{p r ~ 0}\right), \\
G_{H}^{T} \Delta \bar{t}_{i H}^{p r} & =0
\end{aligned}
$$

where $P_{H}$ is an orthogonal projector onto $\operatorname{Ker}\left(G_{H}^{T}\right)$ defined as

$$
P_{H}=I_{H}-G_{H}\left(G_{H}^{T} G_{H}\right)^{-1} G_{H}^{T} .
$$

The FETI algorithm can be regarded as a two-step preconditioned conjugate gradient method to solve the interface problem and can be found abundantly in the literature $[5,6,8,17]$. This algorithm can be summarized as in [6]:

1. Initialize

$$
\begin{aligned}
\bar{t}_{i H}^{p r 0} & =G_{H}\left(G_{H}^{T} G_{H}\right)^{-1} \bar{g}_{i H}^{p r} \\
w^{0} & =P_{H}^{T}\left(\bar{d}_{i H}^{p r}-F_{H} \bar{t}_{i H}^{p r ~}{ }^{0}\right)
\end{aligned}
$$

2. Iterate

$$
\begin{aligned}
y^{n} & =P_{H} \tilde{F}_{H}^{-1} w^{n} \\
z^{n} & =y^{n}-\sum_{i=0}^{n-1} \frac{y^{n T} F_{H} z^{i}}{z^{i T} F_{H} z^{i}} z^{i} \\
\eta^{n} & =\frac{y^{n T} w^{n}}{z^{n T} F_{H} z^{n}} \\
\bar{t}_{i H}^{p r}{ }^{n+1} & =\bar{t}_{i H}^{p r}+\eta^{n} z^{n} \\
w^{n+1} & =w^{n}-\eta^{n} P_{H}^{T} F_{H} z^{n}
\end{aligned}
$$

where $\tilde{F}_{H}^{-1}$ denotes a chosen preconditioner.

The FETI iterations residual obeys the stopping criterion, $\frac{\left\|\tilde{F}_{H}^{-1} w^{n}\right\|_{2}}{\left\|\bar{q}_{i H}^{p r}\right\|_{2}} \leq \epsilon_{g}$, where $\varepsilon_{g}$ is the global FETI tolerance. In this study, $\epsilon_{g}$ is set to $10^{-7}$.

\subsubsection{The hybrid flux associated with the dual problem}

Similarly, the dual hybrid flux problem (38), (39) and (40) can be written in the standard FETI interface problem for dual residuals $\bar{q}_{i H}^{d u(k)}$, which are the residual of the dual problem (33) and (34) on each subdomain $\Omega^{(k)}$ :

$$
\left[\begin{array}{cc}
F_{H} & -G_{H} \\
-G_{H}^{T} & 0
\end{array}\right]\left[\begin{array}{c}
\bar{t}_{i_{H}}^{d u} \\
\bar{\alpha}_{i H}^{d u}
\end{array}\right]=\left[\begin{array}{c}
\bar{d}_{i H}^{d u} \\
-\bar{g}_{i H}^{d u}
\end{array}\right]
$$

where 


$$
\begin{aligned}
& \bar{\alpha}_{i H}^{d u}=\left[\begin{array}{lll}
\bar{\alpha}_{i H}^{d u}(1) & \ldots & \bar{\alpha}_{i H}^{d u}\left(N_{k}\right)
\end{array}\right], \\
& \bar{d}_{i H}^{d u}=\sum_{k=1}^{N_{k}} H_{H}^{(k)} K_{H}^{(k)^{+}} \bar{q}_{i H}^{d u}(k) \\
& \bar{g}_{i H}^{d u}=\left[R_{H}^{(1)^{T}} \bar{q}_{i H}^{d u}(1)\right] \ldots\left[R_{H}^{\left(N_{k}\right)^{T}} \bar{q}_{i H}^{d u}\left(N_{k}\right)\right], \\
& \bar{q}_{i H}^{d u}{ }^{(k)}=-\left[\left(A_{H}^{(k)}+D_{H}^{(k)}\right) \bar{\psi}_{i H}^{(k)}+\sum_{j=1}^{3} E_{j i H}^{(k)}{ }^{T} \bar{\psi}_{j H}^{(k)}+B_{i H}^{(k)}{ }^{T} \bar{\lambda}_{H}^{(k)}\right]-\bar{\ell}_{i H}^{0} .
\end{aligned}
$$

The PCPG algorithm is also used for solving the above FETI interface problem for the three decoupled dual hybrid flux components $\bar{t}_{i H}^{d u}$.

\subsection{Fine mesh calculation}

Before performing the fine mesh calculations for the field errors, the variables $u_{i H}^{(k)}, p_{H}^{(k)}, \psi_{i H}^{(k)}$, $\lambda_{H}^{(k)}, t_{i H}^{p r}$ and $t_{i H}^{d u}$ are interpolated from the coarse $H$-mesh onto the fine $h-$ mesh. The solutions of the primal and dual problems, $u_{i H}^{(k)}$ and $\psi_{i H}^{(k)}$, which satisfy local incompressibility on the coarse $H$-mesh, however, will not satisfy this condition on the fine $h-$ mesh. Incompressible local projectors have to be introduced so that these variables can be modified and local continuity on the fine mesh can still be satisfied. Similar to the coarse mesh calculations, the fine mesh calculations involve the separated primal and dual parameters for the incompressible local projections and reconstructed errors.

\subsection{1 'Incompressible local projections'}

The incompressible local projectors $\left(\Delta_{h}^{p r(k)}, \delta_{h}^{p r(k)}\right)=\left(\Delta_{1 h}^{p r(k)}, \Delta_{2 h}^{p r(k)}, \Delta_{3 h}^{p r(k)}, \delta_{h}^{p r(k)}\right)$ for the primal problem are evaluated by

$$
\begin{aligned}
A_{h}^{(k)} \bar{\Delta}_{i h}^{p r(k)}+B_{i h}^{(k)}{ }^{T} \bar{\delta}_{h}^{p r(k)} & =0, \quad i=1,2,3, \\
\sum_{i=1}^{3} B_{i h}^{(k)} \bar{\Delta}_{i h}^{p r(k)} & =-\sum_{i=1}^{3} B_{i h}^{(k)} \bar{u}_{i H}^{p r(k)} .
\end{aligned}
$$

Then, the field solution can be modified as $\tilde{u}_{i h}^{(k)}=u_{i H}^{(k)}+\Delta_{i h}^{p r(k)}$ and $\tilde{p}_{h}^{(k)}=p_{H}^{(k)}+\delta_{h}^{p r(k)}$, and the incompressible conditions can be satisfied on the fine mesh.

Similarly, the incompressible local projectors, $\left(\Delta_{i h}^{d u^{(k)}}, \delta_{h}^{d u}{ }^{(k)}\right)$, which are introduced for the dual problem, are given by

$$
\begin{aligned}
& A_{h}^{(k)} \bar{\Delta}_{i h}^{d u(k)}+B_{i h}^{(k)^{T}} \bar{\delta}_{h}^{d u(k)}=0, \quad i=1,2,3, \\
& \sum_{i=1}^{3} B_{i h}^{(k)} \bar{\Delta}_{i h}^{d u}(k)=-\sum_{i=1}^{3} B_{i h}^{(k)} \bar{\psi}_{i H}^{d u}{ }^{(k)} .
\end{aligned}
$$

Modifying the associated adjoints by the incompressible projection correctors leads to $\tilde{\psi}_{i h}^{(k)}=$ $\bar{\psi}_{i H}^{(k)}+\Delta_{i h}^{d u(k)}$ and $\tilde{\lambda}_{h}^{(k)}=\lambda_{H}^{(k)}+\delta_{h}^{d u(k)}$. 


\subsection{2 'Reconstructed errors'}

The hybrid flux vectors $\mathbf{t}_{H}^{ \pm}=\kappa \mathbf{t}_{H}^{p r} \pm \mathbf{t}_{H}^{d u}$ are also interpolated to $\mathbf{t}_{h}^{ \pm}=\kappa \mathbf{t}_{h}^{p r} \pm \mathbf{t}_{h}^{d u}$ from the coarse mesh $\mathcal{T}_{H}$ onto the fine mesh $\mathcal{T}_{h}$, and the errors, $\left(\mathbf{e}_{h}^{ \pm(k)}, \varepsilon_{h}^{ \pm(k)}\right)=\left(\mathbf{e}_{h}^{p r(k)}, \varepsilon_{h}^{p r(k)}\right) \pm \frac{1}{\kappa}\left(\mathbf{e}_{h}^{d u^{(k)}}, \varepsilon_{h}^{d u^{(k)}}\right)$, can be determined by the stationarity conditions.

By setting the stationarity conditions (35) and (36) to be satisfied on the fine mesh, the reconstructed errors for the primal problem can be evaluated by the residuals of the associated problem with the primal hybrid flux recovered as Neumann boundary conditions:

$$
\begin{aligned}
& \left.2\left[A_{h}^{(k)} \bar{e}_{i h}^{p r(k)}+B_{i h}^{(k)^{T}} \bar{\varepsilon}_{h}^{p r(k)}\right]=-H_{h}^{(k)^{T}} \bar{t}_{i h}^{p r}-\left[A_{h}^{(k)}+D_{h}^{(k)}\right] \overline{\tilde{u}}_{i h}^{(k)}-B_{i h}^{(k)}\right)^{T} \overline{\tilde{p}}_{h}^{(k)}+\bar{f}_{i h}^{(k)}, \\
& 2 \sum_{i=1}^{3} B_{i h}^{(k)} \bar{e}_{i h}^{p r(k)}=0,
\end{aligned}
$$

and (38) and (39) lead to the errors for the dual problem on the fine mesh:

$$
\begin{aligned}
& 2\left[A_{h}^{(k)} \bar{e}_{i h}^{d u}{ }^{(k)}+B_{i H}^{(k)^{T}} \bar{\varepsilon}_{h}^{d u(k)}\right]=-H_{h}^{(k)^{T}} \bar{t}_{i h}^{d u}-\left[\left(A_{h}^{(k)}+D_{h}^{(k)^{T}}\right] \overline{\tilde{\psi}}_{i h}^{(k)}-B_{i h}^{(k)^{T}} \overline{\tilde{\lambda}}_{h}^{(k)}-\bar{\ell}_{i h}^{0}\right. \\
&\left.-\sum_{j=1}^{3} E_{j i h}^{(k)}\right)^{T} \overline{\tilde{\psi}}_{j h}^{(k)} \\
& 2 \sum_{i=1}^{3} B_{i h}^{(k)} \bar{e}_{i h}^{d u}(k)=0
\end{aligned}
$$

The above fine mesh calculations only involve linear, symmetric systems and can be solved inexpensively by the Conjugate Gradient method with the ILU preconditioner.

\subsection{The bounds and half bound gaps}

Equations (21) and (25) simplify to the upper and lower bounds expressed in matrix form

$$
\begin{gathered}
s_{L B}=\sum_{k=1}^{N_{k}} \sum_{i=1}^{3}\left\{\ell_{i h}^{0} \overline{\tilde{u}}_{i h}^{(k)}+\overline{\tilde{u}}_{i h}^{(k)^{T}}\left(A_{h}^{(k)}+D_{h}^{(k)}\right) \tilde{\tilde{\psi}}_{i h}^{(k)}+\overline{\tilde{p}}_{h}^{(k)^{T}} B_{i h}^{(k)} \tilde{\tilde{\psi}}_{i h}^{(k)}+\overline{\tilde{\lambda}}_{h}^{(k)^{T}} B_{i h}^{(k)} \overline{\tilde{u}}_{i h}^{(k)}-\bar{f}_{h}^{(k)^{T}} \tilde{\bar{\psi}}_{i h}^{(k)}\right. \\
\left.\quad-\kappa \bar{e}_{i h}^{+(k)^{T}} A_{h}^{(k)} \overline{\bar{e}}_{i h}^{+(k)}\right\}, \\
s_{U B}=\sum_{k=1}^{E_{k}} \sum_{i=1}^{3}\left\{\ell_{i h}^{0} \overline{\tilde{u}}_{i h}^{(k)}+\overline{\tilde{u}}_{i h}^{(k)}\left(A_{h}^{(k)}+D_{h}^{(k)}\right) \overline{\tilde{\psi}}_{i h}^{(k)}+\overline{\tilde{p}}_{h}^{(k)^{T}} B_{i h}^{(k)} \overline{\tilde{\psi}}_{i h}^{(k)}+\overline{\tilde{\lambda}}_{h}^{(k)} B_{i h}^{(k)} \overline{\tilde{u}}_{i h}^{(k)}-\bar{f}_{h}^{(k)^{T}} \overline{\tilde{\psi}}_{i h}^{(k)}\right. \\
\left.+\kappa \bar{e}_{i h}^{-(k)^{T}} A_{h}^{(k)} \bar{e}_{i h}^{-(k)}\right\} .
\end{gathered}
$$

Note that the term $\sum_{k=1}^{N_{k}} \sum_{i=1}^{3}\left[\overline{\tilde{u}}_{i h}^{(k)^{T}}\left(A_{h}^{(k)}+D_{h}^{(k)}\right) \overline{\tilde{\psi}}_{i h}^{(k)}+\overline{\tilde{p}}_{h}^{(k)^{T}} B_{i h}^{(k)} \overline{\tilde{\psi}}_{i h}^{(k)}+\overline{\tilde{\lambda}}_{h}^{(k)^{T}} B_{i h}^{(k)} \overline{\tilde{u}}_{i h}^{(k)}-\bar{f}_{h}^{(k)^{T}} \overline{\tilde{\psi}}_{i h}^{(k)}\right]$ is very small compared with the bound gap, $\Delta\left(\mathcal{T}_{\mathcal{H}}, \kappa\right) \equiv \frac{1}{2}\left(s_{U B}-s_{L B}\right)$. Therefore, the modified linear output of interest, $\overline{\tilde{s}}_{H}$, is defined as

$$
\overline{\tilde{s}}_{H}=\sum_{k=1}^{N_{k}} \sum_{i=1}^{3}\left\{\bar{\ell}_{i h}^{0} \overline{\tilde{u}}_{i h}^{(k)}+\overline{\tilde{u}}_{i h}^{(k)^{T}}\left(A_{h}^{(k)}+D_{h}^{(k)}\right) \overline{\tilde{\psi}}_{i h}^{(k)}+\overline{\tilde{p}}_{h}^{(k)^{T}} B_{i h}^{(k)} \overline{\tilde{\psi}}_{i h}^{(k)}+\overline{\tilde{\lambda}}_{h}^{(k)^{T}} B_{i h}^{(k)} \overline{\tilde{u}}_{i h}^{(k)}-\bar{f}_{h}^{(k)^{T}} \overline{\tilde{\psi}}_{i h}^{(k)}\right\} .
$$

Finally, the lower and upper bounds have the simple expression as follows: 


$$
\begin{aligned}
& s_{L B}=\tilde{s}_{H}-\kappa \sum_{k=1}^{N_{k}} \sum_{i=1}^{3} \bar{e}_{i h}^{+(k)^{T}} A_{h}^{(k)} \bar{e}_{i h}^{+(k)}, \\
& s_{U B}=\tilde{s}_{H}+\kappa \sum_{k=1}^{N_{k}} \sum_{i=1}^{3} \bar{e}_{i h}^{-(k)^{T}} A_{h}^{(k)} \bar{e}_{i h}^{-(k)} .
\end{aligned}
$$

The half bound gap is:

$$
\Delta\left(\mathcal{T}_{\mathcal{H}}, \kappa\right) \equiv \frac{1}{2}\left(s_{U B}-s_{L B}\right)=\sum_{k=1}^{N_{k}} \sum_{i=1}^{3}\left[\kappa \bar{e}_{i h}^{p r(k)^{T}} A_{h}^{(k)} \bar{e}_{i h}^{p r(k)}+\frac{1}{\kappa} \bar{e}_{i h}^{d u(k)^{T}} A_{h}^{(k)} \bar{e}_{i h}^{d u}(k)\right] .
$$

The output predictor is therefore rewritten in matrix form as

$$
s_{\text {pre }}\left(\mathcal{T}_{H}\right)=\tilde{s}_{H}-2 \sum_{k=1}^{N_{k}} \sum_{i=1}^{3} \bar{e}_{i h}^{p r(k)^{T}} A_{h}^{(k)} \bar{e}_{i h}^{d u(k)} .
$$

Note that equation (74) is independent of the parameter $\kappa$.

\subsection{Optimal bounds}

Because the calculations of $\left(\mathbf{e}_{h}^{p r(k)}, \varepsilon_{h}^{p r}\right)$ and $\left(\mathbf{e}_{h}^{d u}(k), \varepsilon_{h}^{d u}\right)$ are not associated with the parameter $\kappa$, the half bound gap (73) can be optimized by choosing optimum $\kappa^{*}$ so that the lower and upper bounds are as sharp as possible [11]:

$$
\left.\frac{\partial \triangle\left(\mathcal{T}_{H}, \kappa\right)}{\partial \kappa}\right|_{\kappa=\kappa^{*}}=\sum_{k=1}^{N_{k}} \sum_{i=1}^{3}\left(\bar{e}_{i h}^{p r(k)}\right)^{T} A_{h}^{(k)} \bar{e}_{i h}^{p r(k)}-\frac{1}{\kappa^{* 2}} \sum_{k=1}^{N_{k}} \sum_{i=1}^{3}\left(\bar{e}_{i h}^{d u(k)}\right)^{T} A_{h}^{(k)} \bar{e}_{i h}^{d u(k)}=0,
$$

which leads to $\kappa^{*}=\sqrt{\frac{\sum \sum \bar{e}_{i h}^{d u(k)^{T}} A_{h}^{(k)} \bar{e}_{i h}^{d u(k)}}{\sum \sum \bar{e}_{i h}^{p r(k)^{T}} A_{h}^{(k)} \bar{e}_{i h}^{p r(k)}}}$.

\section{Numerical Results on a Single Processor}

In this section, numerical results on one processor are reported for bounds to the output of the steady, incompressible, low Reynolds number Navier-Stokes equations. The linear output $s_{h}$ associated with the fine discretization $\mathcal{T}_{h}$ is bounded by the upper and lower bounds, $s_{U B}$ and $s_{L B}$. The predicted linear output is the average of the upper and lower bounds, i.e., $s_{\text {pre }}=\frac{1}{2}\left(s_{U B}+s_{L B}\right)$. All the computations are carried on a single processor Pentium III $933 \mathrm{MHz}$ CPU with 2 Gigabyte RAM memory, running SUN Solaris Operating System 5.8. Computational results are presented here to illustrate the accuracy of the bound method.

The domain is decomposed into $3 \times 3 \times 3-3=24$ uniform subdomains, each of which is a cube of size $\frac{1}{3} \times \frac{1}{3} \times \frac{1}{3}$. The fine mesh is assumed to be $\mathcal{T}_{h=0.02778}$, representing the discretizations of the domain into $[(36 \times 36 \times 36)-3 \times(12 \times 12 \times 12)] \times 6=248,832$ tetrahedra. This problem has more than 4 million degrees-of-freedom (d.o.f.). It is impossible for a $2 \mathrm{G}$ memory computer to simulate such a problem by the standard finite element method unless some virtual memory is used. The bound method, however, can predict the output of this 4 million d.o.f. problem with the stated computer resources. Five different coarse subdivisions are considered for the N-S problem with 24 subdomain decomposition: i.e., $\mathcal{T}_{0.05556}, \mathcal{T}_{0.08333}, \mathcal{T}_{0.11111}, \mathcal{T}_{0.16667}$, and $\mathcal{T}_{0.33333}$, representing the uniform coarse discretizations of the global domain divided into $24 \times(4 \times 4 \times 4) \times 6=9,216,24 \times(3 \times 3 \times 3) \times 6=3,888,24 \times(2 \times 2 \times 2) \times 6=1,152$ and $24 \times(1 \times 1 \times 1) \times 6=144$ tetrahedra, respectively. Note that the coarsest mesh, $\mathcal{T}_{0.33333}$, is 
the coarsest structured mesh that can constructed for this geometry. It will be used to test our hypothesis that the bounds are in the asymptotic certainty regime. to These coarse subdivisions are further refined into the 'truth' mesh for the local Neumann calculations, namely, $\mathcal{T}_{0.02778}$, with $R=3,4,6,9,12$, respectively. The reader is referred to Table 1 for the number of elements and the degrees-of-freedom associated with each refinement $R$ investigated for this problem.

\begin{tabular}{|c|c|c|c|c|c|c|c|c|}
\hline \multicolumn{4}{|c|}{ Coarse mesh } & Memory & \multicolumn{2}{c|}{ CPU (k sec.) } & \multicolumn{2}{c|}{$\Delta\left(\mathcal{T}_{H}, \kappa^{*}\right) \%$} \\
\hline $\mathcal{T}_{H}$ & elements & d.o.f. & $R$ & M bytes & $R e=1$ & $R e=100$ & $R e=1$ & $R e=100$ \\
\hline $\mathcal{T}_{0.05556}$ & 31,104 & 519,444 & 2 & 1552 & 684 & 776 & 4.211 & 8.481 \\
\hline $\mathcal{T}_{0.08333}$ & 9,216 & 157,380 & 3 & 484 & 130 & 135 & 6.521 & 13.495 \\
\hline $\mathcal{T}_{0.11111}$ & 3,888 & 67,932 & 4 & 350 & 103 & 101 & 8.752 & 18.326 \\
\hline $\mathcal{T}_{0.16667}$ & 1,152 & 21,108 & 6 & 308 & 95 & 103 & 13.952 & 31.596 \\
\hline $\mathcal{T}_{0.33333}$ & 144 & 3,084 & 12 & 308 & 104 & 100 & 39.376 & 82.783 \\
\hline
\end{tabular}

Table 1: Subdivisions, refinements, computer resources and half bound gaps for $R e=1$ and $R e=100$.

For the very low Reynolds number problems, $R e \ll 1$, the convection term is very weak compared with the diffusion term, indicating that the Stokes bound procedure can be used to solve the underlying Navier-Stokes bound problem [3]. So in this paper, the investigation is focused on $R e \in[1,100]$. Recall that the Reynolds number represents the applied pressure gradient.

ag replacements
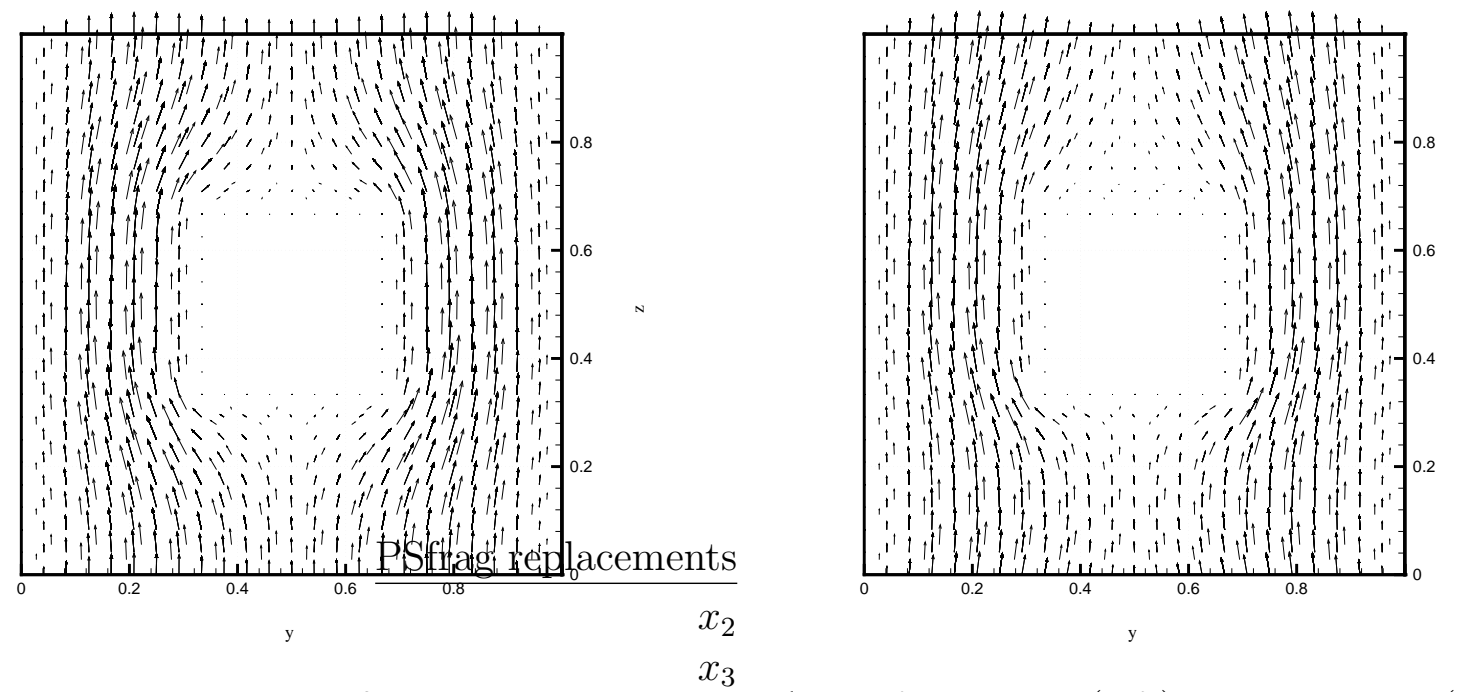

$x_{3}$

$x_{3}$

Figure 4: The velocity profile on the coarse mesh $\mathcal{T}_{0.08333}$ for $R e=1$ (left) and $R e=100$ (right).

Figure 4 shows the velocity profiles for the Navier-Stokes problem with the Reynolds numbers $R e=1$ and $R e=100$, respectively, at section $x_{1}=0.5$. The driving force $f_{1}=f_{2}=0.0$ and $f_{3}=\frac{28.45}{R e}$ are selected for the different Reynolds numbers.

It is very expensive to calculate the output $s_{h}$ because the truth $h-$ mesh problem has more than 4 million d.o.f., the predicted output $s_{\text {pre }}$ can be used as an alternative to $s_{h}$. Therefore, the relative bounds are denoted by $s_{U B} / s_{\text {pre }}, s_{L B} / s_{\text {pre }}$, and the relative coarse mesh output is denoted by $s_{H} / s_{\text {pre }}$ as a function of $H$. Figure 5 shows that the optimized bound gaps converge with respect to the coarse mesh for different Reynolds numbers. For $R e=1$, the optimized 
ag replacements

Relative Bounds

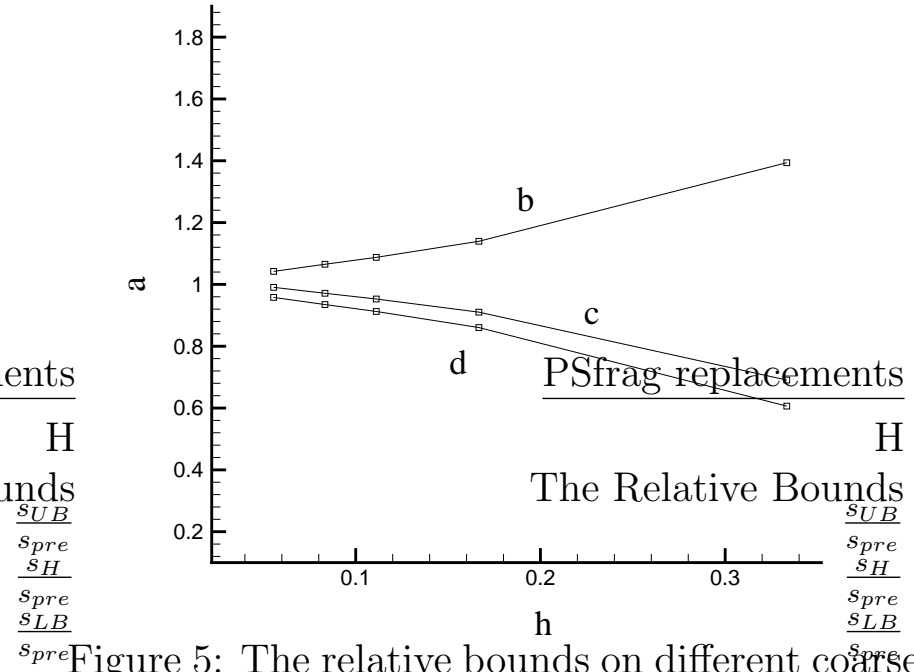

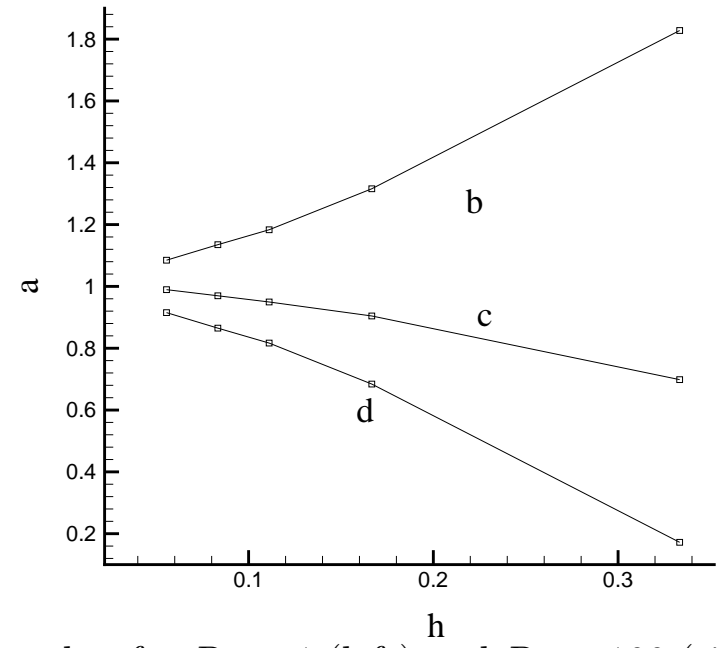

$\mathrm{h}$

${ }^{s_{p r e}}$ Figure 5: The relative bounds on different cosmerse meshes for $R e=1$ (left) and $R e=100$ (right).

half bound gap is less than $39.376 \%$ for the coarsest mesh $\mathcal{T}_{0.333333}$ and decreases to less than $4.211 \%$ for $\mathcal{T}_{0.05556}$. For $R e=100$, the optimized half bound gap decreases from $82.783 \%$ to $8.481 \%$ with the reduction of the mesh size from $\mathcal{T}_{0.333333}$ to $\mathcal{T}_{0.05556}$.

ag replacements

$e_{H}=\left|s_{H}-\breve{s}_{H}\right|$

${ }_{B}=\left|s_{U B}-\breve{s}_{U B}\right|$

$B=\left|s_{L B}-\breve{s}_{L B}\right|$

re $=\left|s_{\text {pre }}-\breve{s}_{\text {pre }}\right|$ , $\log e_{L B}, \log e_{\text {pre }}$
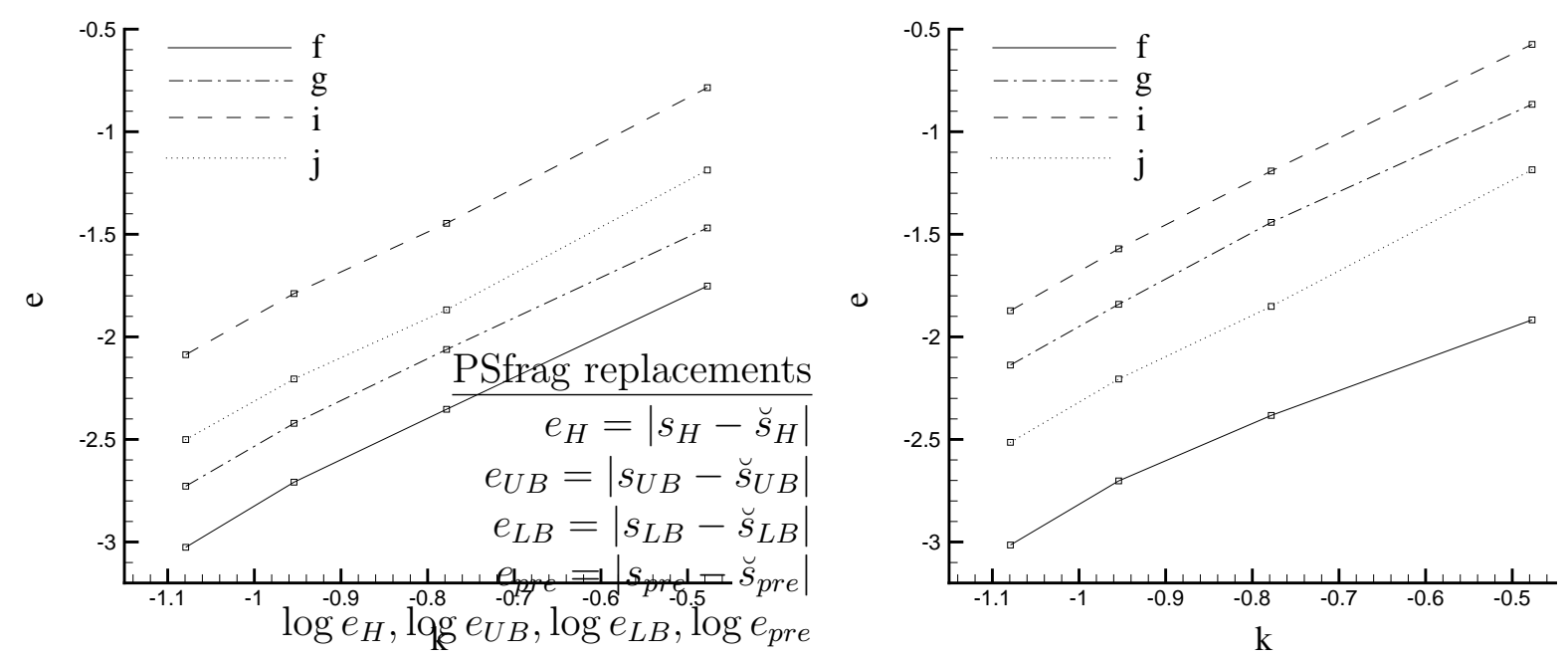

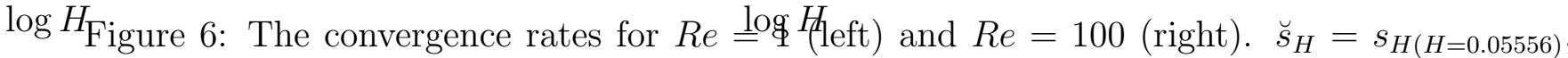
$\breve{s}_{U B}=s_{U B(H=0.05556)}, \breve{s}_{L B}=s_{L B(H=0.05556)}, \breve{s}_{\text {pre }}=s_{\text {pre }(H=0.05556)}$.

Figure 6 presents the convergence rates of the coarse mesh output $e_{H}$, the bounds $e_{U B}$, $e_{L B}$, and the predicted value $e_{\text {pre }}$ as a function of $\log H$ on a $\log -\log$ scale. $\breve{s}_{H}, \breve{s}_{U B}, \breve{s}_{L B}$ and $\breve{s}_{\text {pre }}$ denote the values of $s_{H}, s_{U B}, s_{L B}$ and $s_{\text {pre }}$ obtained at $H=0.05556$, respectively. For the different Reynolds numbers, the slopes of $e_{H}, e_{p r e}, e_{U B}$ and $e_{L B}$ are about $O\left(H^{2.1}\right)$, $O\left(H^{2.2}\right), O\left(H^{2.2}\right)$ and $O\left(H^{2.1}\right)$, respectively. The fact that these convergence curves are smooth ensure that the bounds are in the asymptotic certainty regime even for our coarsest mesh. The characteristic size of this mesh is one third of the computational size and can be considered very large, nevertheless, as it can be seen from the above results, this size is still less than the threshold mesh size required to obtain full certainty. 
Table 1 also shows the computational efficiency for the Navier-Stokes bound method. The optimized relative half bound gap, the memory and the simulation time which are associated with the different coarse mesh sizes are reported in this table. The bound method carried out on a coarse mesh size $\mathcal{T}_{0.05556}$ uses $776 \mathrm{k}$ seconds CPU time and $1552 \mathrm{M}$ memory to predict the linear output of 4 million d.o.f. problem with an error less than $8.5 \%$ for $R e=100$. This Reynolds number is equivalent to a Reynolds number based on the average velocity of approximately 20. If the Reynolds number is increased, separation occurs and the coarse meshes used in the present study become unacceptable, i.e., the bound gap is greater than $500 \%$ for $R e=300$. Clearly, the coarse mesh must be refined to capture all features of this flow at higher Reynolds numbers.

\section{Parallel Results from a Beowulf Cluster}

In the previous section it was shown that the bound method offers a significant computational advantage over the standard finite element method. To be noted, for one processor numerical simulations on meshes coarser than $\mathcal{T}_{0.08333}$, the largest amount of computational time is spent on the fine mesh calculations, which require $73.4 \%$ to $99.94 \%$ of the total CPU because the coarse mesh problems have very few d.o.f. compared with the fine mesh ones. These decoupled fine mesh calculations are nevertheless much less expensive because they actually involve linear algebraic systems, just like those for the Stokes equations. Parallelization of the fine mesh calculations can further improve the computational efficiency of the bound method.

Indeed, the fine mesh calculations are intrinsically parallel because they are completely decoupled problems. Therefore, subdomain problems can be calculated simultaneously on different processors with little communication between them, translating into large parallel efficiency. Generally, the bound method achieves very high efficiencies (more than 90\%) for the fine mesh local calculations even on a Beowulf cluster. The only parallel efficiency loss comes from the different CPU time used for iteratively solving the local subdomain problems on different processors. On some subdomains, the iterative solver may converge quickly, whereas on others it may take a longer time. The slight difference in the convergence time leads to fine mesh calculations that do not finish at the same time, and therefore less than perfect $100 \%$ parallel efficiency. This can be effectively improved by dynamically loading the jobs on different processors.

The coarse mesh FETI calculations are performed by an efficient parallel semi-iterative solver. The cost is associated with the calculation of the coarse interface problem. In each FETI outer loop iteration, the number of degrees-of-freedom is equal to the total number of subdomains, in this case, 24 subdomains. This leads to a very small global system. The inner loop iteration involves local systems and can be easily parallelized. Parallel efficiency of the FETI method is theoretically and numerically demonstrated in the literature [6, 7]. The above characteristics suggest the high potential of extending the bound method with the FETI procedure to parallel computations.

Parallel results reported here are obtained on a Beowulf cluster of Pentium III 933MHz/1024M memory computers, connected with a 10/100 Mbits/sec hub, running the SunOS 5.8 operating system. The communication uses the Message Passing Interface (MPI) available through the MPICH library.

Table 2 reports the performance results for the increasing number of processors for $R e=1$ 


\begin{tabular}{|c|c|c|c|c|c|c|c|c|}
\hline Proc. & \multicolumn{4}{|c|}{ Elapsed Time $(\mathrm{sec})$ on $\mathcal{T}_{0.08333}$} & \multicolumn{3}{c|}{ Parallel Efficiency $\mathcal{T}_{0.08333}$} \\
\hline$\#$ & Coarse & FETI & Fine Cal. & Total & FETI & Fine & Total & Speedup \\
\hline 1 & $27.4 \mathrm{k}$ & $7.1 \mathrm{k}$ & $95.4 \mathrm{k}$ & $129.9 \mathrm{k}$ & $/$ & $/$ & $/$ & $/$ \\
\hline 2 & $27.4 \mathrm{k}$ & $3.9 \mathrm{k}$ & $48.2 \mathrm{k}$ & $79.4 \mathrm{k}$ & $92.1 \%$ & $99.0 \%$ & $81.8 \%$ & 1.63 \\
\hline 3 & $27.4 \mathrm{k}$ & $2.7 \mathrm{k}$ & $32.2 \mathrm{k}$ & $62.3 \mathrm{k}$ & $89.3 \%$ & $98.6 \%$ & $69.5 \%$ & 2.08 \\
\hline 4 & $27.4 \mathrm{k}$ & $2.2 \mathrm{k}$ & $25.3 \mathrm{k}$ & $54.8 \mathrm{k}$ & $82.1 \%$ & $98.9 \%$ & $59.2 \%$ & 2.37 \\
\hline 6 & $27.4 \mathrm{k}$ & $1.5 \mathrm{k}$ & $16.0 \mathrm{k}$ & $44.9 \mathrm{k}$ & $80.1 \%$ & $99.1 \%$ & $48.2 \%$ & 2.89 \\
\hline 8 & $27.4 \mathrm{k}$ & $1.1 \mathrm{k}$ & $12.1 \mathrm{k}$ & $40.6 \mathrm{k}$ & $79.2 \%$ & $98.6 \%$ & $40.0 \%$ & 3.20 \\
\hline
\end{tabular}

Table 2: Performance results, parallel efficiency and speedup for increasing number of processors with $R e=1$ on $\mathcal{T}_{0.08333}$

and the coarse mesh of $\mathcal{T}_{0.08333}$, as well as their corresponding parallel efficiencies: $81.8 \%$ for 2 processors and $69.5 \%$ for 3 processors. Note that the global coarse mesh calculations for the primal and dual problems are not parallelized so the CPU time spent on these two steps are always about $27.4 \mathrm{k}$ seconds no matter how many processors are used. This is the reason that the total parallel efficiency is lower than those of both FETI and fine mesh calculations. We can see that the efficiency for small number of processors is satisfactory, but it becomes poor for a large number of computers (more than 4 processors). The nonparallelization of the global coarse problems in the bound procedure reduces the total efficiency from $59.2 \%$ on 4 processors to $40 \%$ on 8 processors.

However, on very coarse meshes, the total parallel efficiency is still as high as $90 \%$, while yielding a satisfactory half bound gap. Table 3 reports the total parallel efficiencies on 8 processors for the N-S problem on different coarse meshes with $R e=1$. The bound method can achieve a half bound gap with $8.752 \%$ error and a total of $75.9 \%$ parallel efficiency on the coarse mesh of $\mathcal{T}_{0.11111}$. On a coarse mesh of $\mathcal{T}_{0.16667}$, the bound method obtains the results with less than $14.0 \%$ error and more than $90 \%$ efficiency for $R e=1$. Note that in all those cases, the output that is bounded is related to the fine mesh $\mathcal{T}_{0.02778}$.

\begin{tabular}{|c|c|c|c|}
\hline \multicolumn{2}{|c|}{ Mesh vs. error } & \multicolumn{2}{c|}{ Efficiency } \\
\hline $\mathcal{T}_{H}$ & $S_{\text {gap }} \%$ & Total (\%) & Speed up \\
\hline $\mathcal{T}_{0.08333}$ & $6.521 \%$ & $40.0 \%$ & 3.20 \\
\hline $\mathcal{T}_{0.11111}$ & $8.752 \%$ & $75.9 \%$ & 6.07 \\
\hline $\mathcal{T}_{0.16667}$ & $13.952 \%$ & $95.8 \%$ & 7.82 \\
\hline $\mathcal{T}_{0.33333}$ & $39.376 \%$ & $98.7 \%$ & 7.90 \\
\hline
\end{tabular}

Table 3: Parallel efficiency and speedup for the different coarse mesh Navier-Stokes problem with $R e=1$ on 8 processors.

The numerical results demonstrate that the total parallel efficiency of the three dimensional bound procedure is close to that of the last step fine mesh Neumann calculations when the very coarse mesh $\mathcal{T}_{H}$ is selected. It proves that the bound procedure with the FETI method is an ideal algorithm with low computational cost, high accuracy and high parallel efficiency. However, a more efficient parallel implementation is nevertheless required and should be developed for large-scale engineering application in the near future. In fact, for the Navier-Stokes equations, the coarse problem should also be calculated in parallel on multi-processors. 


\section{Conclusion}

The a posteriori finite element bound method efficiently evaluates the 'truth' mesh output by calculating upper and lower bounds to this output, and thus provides error information related to this output. The augmented Lagrangian based on a coarse mesh linearization of the N-S equations and on a domain decomposition approach leads to a simplified bound procedure for the three dimensional Navier-Stokes equations. Numerical results for the problem of interest show that the resulting bounds are sharp, rigorous, and computational resources can be significantly saved. It is also shown herein that the uncertainty due to coarse mesh size is mainly a theoretical concern and that, in practice, rigorous bounds are obtained for very coarse meshes. The cost overhead of the bound method is small. It is obvious that by comparing the coarse mesh calculations to solve the field velocity and pressure $\left(\mathbf{u}_{\mathbf{H}}, p_{H}\right)$ with the total cost of calculating both the upper and lower bounds, e.g., $27.4 \mathrm{k}$ seconds vs. $130 \mathrm{k}$ seconds on $\mathcal{T}_{0.08333}$ for $R e=1$, the bound method provides certainty information for a low additional cost. The bound method has the potential to be an inexpensive and reliable approach to incorporate numerical simulation of large-scale engineering problems in engineering design.

\section{Acknowledgements}

This work was supported by the Natural Sciences and Engineering Research Council of Canada (NSERC).

\section{References}

[1] M. Ainsworth and J. T. Oden. A unified approach to a posteriori error estimation using element residual methods. Numer. Math., 65:23-50, 1993.

[2] R. E. Bank and B. D. Welfert. A posteriori error estimates for the Stokes problem. SIAM J. Numer. Anal., 28:591-623, 1991.

[3] Z. Cheng. A Posteriori Finite Element Bounds: Application to Outputs of the Three Dimensional Navier-Stokes Equations. PhD thesis, Graduate Department of Mechanical and Industrial Engineering, University of Toronto, April 2003.

[4] Z. Cheng and M. Paraschivoiu. Parallel Computions of Finite Element Output Bounds for Conjugate Heat Tranfer. Finite Element in Analysis and Design, 39/7:581-597, 2003.

[5] C. Farhat, P.-S. Chen, F. Risler, and F.-X. Roux. A unified framework for accelerating the convergence of iterative substructuring methods with Lagrange multipliers. Int. J. Numer. Meth. Engrg., 42:257-288, 1998.

[6] C. Farhat, K. Pierson, and M. Lesoinne. The second generation FETI methods and their application to parallel solution of the large-scale linear and geometrically non-linear structural analysis problems. Comput. Methods Appl. Mech. and Engrg., 184:333-374, 2000.

[7] C. Farhat and F.-X. Roux. A method of finite tearing and interconnecting and its parallel solution algorithm. Comput. Methods. Appl. Mech. Engrg., 32:1205-1227, 1991. 
[8] C. Farhat and F.-X. Roux. An unconventional domain decomposition method for efficient parallel solution of large-scale finite element systems. SIAM J. Sci. Stat. Comput., 13(1):379-396, 1992.

[9] M. D. Gunzberger. Finite Element Methods for Viscous Incompressible Flows, A Guide to Theory, Practice, and Algorithms. Academic Press, San Diego, 1989.

[10] P. Ladeveze and D. Leguillon. Error esimation procedures in the finite element method and applications. SIAM J. Numer. Anal., 20:485-509, 1983.

[11] L. Machiels, J. Peraire, and A. T. Patera. A Posteriori Finite Element Output Bounds for the Incompressible Navier-Stokes Equations: Application to a Natural Convection Problem. Journal of Computational Physics, 172:401-425, 2001.

[12] Y. Maday, A. T. Patera, and J. Peraire. A general formulation for a posteriori bounds for output functionals of partial differential equations; application to the eighenvalue problem. C. R. Acad. Sci., Paris, Serie I, 328:828-836, 1999.

[13] Y. Maday and A.T. Patera. Numerical Analysis of a posteriori Finite Element Bounds for Linear-Functional Outputs. Math. Models and Meth. in Appl. Sci., 10 (5):785-799, 2000.

[14] Bruce R. Munson, Donald F. Young, and Theodore H. Okiishi. Fundamentals of Fluid Mechanics. John Wiley \& Sons, New York, 1990.

[15] J. T. Oden, W. Wu, and M. Ainsworth. An A Posteriori Error Estimate for Finite Element Approximations of the Navier-Stokes Equations. TICOM Report 93-05, 1993.

[16] M. Paraschivoiu. A Posteriori Finite Element Bounds for Linear-Functional Outputs of Coericive Partial Differential Equations and of the Stokes Problems. PhD thesis, Department of Mechanical Engineering, Massachusetts Institute of Technology, October 1997.

[17] M. Paraschivoiu. A Posteriori finite element output bounds in three space dimensions using the FETI method. Comput. Methods Appl. Mech. and Engrg., 190 (49-50):6629-6640, 2001.

[18] M. Paraschivoiu, J. Peraire, and A. T. Patera. A Posteriori finite element bounds for linearfunctional outputs of elliptic Partial Differential Equations. Comput. Methods Appl. Mech. and Engrg., 150:289-312, 1997.

[19] A. Quarteroni and A. Valli. Numerical Approximation of Partial Differential Equation. Springer-Verlag, 1994.

[20] R. Temam. Navier Stokes Equations: Theory and Numerical Analysis. North-Holland Publishing, Amsterdam, 1979.

[21] R. Verfürth. A posteriori error estimators for the Stokes equations. Numer. Math., 55:309325, 1989.

[22] O. C. Zienkiewicz, Y. C. Liu, and G. C. Huang. Error estimates and convergence rates for various incompressible elements. Intl. J. Num. Meth. Eng., 28:2191-2202, 1989. 